\title{
VERA observations of SiO maser emission from R Aquarii
}

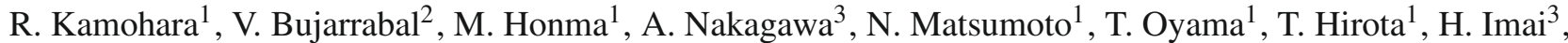 \\ K. M. Shibata ${ }^{1}$, H. Kobayashi ${ }^{1}$, K. Sato ${ }^{4}$, and Y. Ueno ${ }^{4}$ \\ 1 Mizusawa VLBI Observatory, National Astronomical Observatory of Japan, 2-21-1 Osawa, Mitaka, Tokyo 181-8588, Japan \\ e-mail: ryuichi.kamohara@nao.ac.jp \\ 2 Observatorio Astronómico Nacional. Ap 112, 28803 Alcalá de Henares, Spain \\ e-mail: v.bujarrabal@oan.es \\ 3 Graduate School of Science and Engineering, Department of Physics, Faculty of Science, Kagoshima University, 1-21-35 Korimoto, \\ Kagoshima 890-0065, Japan \\ 4 Mizusawa VLBI Observatory, National Astronomical Observatory of Japan, 2-12 Hoshi-ga-oka, Mizusawa-ku, Oshu-shi, \\ Iwate 023-0861, Japan
}

Received 7 May 2009 / Accepted 5 November 2009

\begin{abstract}
Aims. We aim to study the spatial distribution of the $v=1$ and $v=2 J=1-0 \mathrm{SiO}$ maser emission from $\mathrm{R}$ Aqr, a well known stellar symbiotic system. In particular, we intend to determine the annual parallax and proper motion of the source by means of measurements of the absolute coordinates of the maser spots.

Methods. We performed VLBI observations of the $v=1$ and $v=2 J=1-0$ maser emission, at 7 mm wavelength, using VERA. We present observations made in 11 epochs, between December 2004 and October 2006, and observations by other authors are also discussed. VERA provides very high spatial resolution and accurate astrometric measurements, thanks to its double-beam observing system. From fitting ring-like structures to the maser spot distributions, we determine absolute J2000 coordinates of the central star. ( $\mathrm{SiO}$ maser emission is known to be distributed around the star forming spot rings at a few stellar radii.)

Results. Maps with accurate absolute coordinates were obtained in 8 epochs. From the coordinates determined for the central star, we measured parallax and linear proper motion. We obtain $\pi=4.7 \pm 0.8$ mas, compatible with, but much more accurate than, the Hipparcos value $\left(5.07 \pm 3.15\right.$ mas), and deduce a distance of R Aqr $D=214_{-32}^{+45} \mathrm{pc}$. Our accurate astrometry also yields a reliable comparison between the spot distributions of both $v=1$ and $v=2 J=1-0$ lines. We find that both masers come from similar regions, i.e. at similar distances from the star and defining common spot clusters, confirming previous results. But the coincidences between spots of both lines are very rare, within the spatial and spectral resolution of our experiments. This result is found for eight epochs, spanning more than one pulsation cycle (of the cool Mira-type component). We argue that explaining the finer details of the $v=1,2 J=1-0$ distributions found here would require new theoretical efforts. Finally, our observations also allow the study of the structure and dynamics of the close circumstellar shells in R Aqr. We do not confirm the previous suggestion that these shells are rotating; instead, we conclude that the observed kinematics is very probably caused by pulsations and random movements. The spatial distribution of the maser spots is found to be variable, but to show a stable axial symmetry.
\end{abstract}

Key words. radio lines: stars - masers - techniques: interferometric - stars: AGB and post-AGB - circumstellar matter stars: individual: R Aqr

\section{Introduction}

$\mathrm{R}$ Aquarii is a symbiotic stellar system including an M-type Mira-like variable and a hot dwarf star. The system presents a high-excitation precessing jet detected in several wavelengths at PA ranging between $\sim 0$ and 45 degrees (e.g. Michalitsianos et al. 1988; Kellogg et al. 2007). A wide nebulosity is also detected in optical atomic lines, which extends about 2 arcmin, mostly in the east-west direction, and seems to be in expansion at about $50 \mathrm{~km} \mathrm{~s}^{-1}$ (e.g. Hollis et al. 1999).

Usually, the nebulae around symbiotic stars are poor in molecular line emission (e.g. Bujarrabal \& Pardo 2007; Ivison et al. 1995). R Aqr is the symbiotic system presenting the beststudied molecular spectra. Its $\mathrm{SiO}$ maser intensity is normal, compared to that of standard Mira stars (Bujarrabal et al. 1987; Pardo et al. 2004) and has been studied relatively well, including high-resolution mapping using VLBI techniques. The SiO maser probably originates in the close surroundings of the Mira-type variable, forming the typical ring of spots (e.g. Boboltz et al. 1997; Hollis et al. 1990).

The orbital parameters of the binary system are still controversial. The two components have been resolved in observations by Hollis et al. (1997), who conclude that the projection of the orbit axis on the plane of the sky presents a PA $\sim 0^{\circ}$ (the direction of the line of the nodes being placed at about 90 degrees, roughly in the plane of optical nebulosity). The orbit is characterized by a distance between stars of about $2 \times 10^{14} \mathrm{~cm}$ and a period of about 44 yr (Hollis et al. 1997; McIntosh \& Rustan 2007). The direction of the orbit axis is, however, controversial due to difficult interpretation of the data and the uncertainty in the distance; see recent discussion by Gromadzki \& Mikołajewska (2009), who propose that the orbit axis is mostly parallel to the optical jets. The Hipparcos parallax is not accurate, and yields values of the distance ranging between 120 and $520 \mathrm{pc}$.

Theoretical models can explain the existence and most properties of $\mathrm{SiO}$ maser emission, although some aspects of the 
$\mathrm{SiO}$ maser formation are still under discussion. In most pumping schemes, the inversion of the rotational transitions in the vibrational state $v$ appears when the $v \rightarrow v-1$ rovibrational transitions are optically thick, yielding significant photon trapping. Two kinds of models, collisional or radiative, exist; the difference is how, collisionally or radiatively, the excited $v$ state is populated from the $v=0$ ground state (see e.g. Bujarrabal \& Nguyen-Q-Rieu 1981; Lockett \& Elitzur 1992).

The spatial structure of the $\mathrm{SiO}$ maser emission from evolved stars is in general explained well. In particular, it is today widely accepted that the ring-like spot distributions, observed in wellmapped $\mathrm{SiO}$ maser lines (see Cotton et al. 2006, and references therein), stem from predominantly tangential amplification, as originally predicted by the radiative mechanisms many years before the first VLBI observations were performed. However, some observational features remain controversial. For instance, the spots of the maser transitions $v=1$ and $v=2 J=1-0$ often occupy similar regions, but are very rarely coincident (Desmurs et al. 2000). This property is not understood well and has been used as an argument for radiative or collisional pumping mechanisms (see details in the extensive discussion by Desmurs et al. 2000, and in Sect. 4.2).

In this paper, we report observational results of $\mathrm{SiO}$ masers toward R Aqr obtained from very accurate astrometric measurements using VERA (VLBI Exploration of Radio Astrometry). We can estimate the distance to the star from measurements of the annual parallax, since the absolute coordinates of the $\mathrm{SiO}$ spots are accurately measured. Thanks to these accurate determinations of absolute coordinates, we are also able to quantititatively study the degree of spot coincidence between $v=1$ and $v=2 J=1-0$ masers. We also conclude that in R Aqr, perhaps due to the binary nature of the object, the structure of the maser spots around the central star is variable and often very peculiar.

We present our observations in Sect. 2 and our estimates of the distance and proper motion in Sect. 3. We also discuss the spot coincidence and the theoretical interpretation of our results (Sect. 4) and the general properties of the spot distribution in this source (Sect. 5). Finally, a summary of our conclusions is given in Sect. 6.

\section{Observations and data reduction}

We have been performing $43 \mathrm{GHz}$ VLBI observations of R Aqr from December 2004, using the 4 antennas of VERA. A unique feature of VERA is its dual-beam system, in which two independent receivers are located at the focal plane of each cassegrain antenna. This dual-beam system allows us to observe two nearby sources (target maser and reference extragalactic source with well-known accurate coordinates, within $2^{\circ} .2$ separation), so as to cancel tropospheric fluctuations out. The instrumental delay difference between the two beams can be calibrated by correlating random signals from artificial noise sources injected onto the receivers (Honma et al. 2008a).

The positional information obtained by VERA is therefore a relative position with respect to a reference source. If the true positions of the target and reference sources are $P_{\mathrm{s}}$ and $P_{\mathrm{r}}$, respectively, and the nominal or adopted coordinates of the target and reference sources are $P_{\mathrm{s}}^{\prime}$ and $P_{\mathrm{r}}^{\prime}$, a VERA observational position offset $P_{\mathrm{s}}^{\prime \prime}$ is

$P_{\mathrm{s}}^{\prime \prime}=P_{\mathrm{s}}-P_{\mathrm{s}}^{\prime}-\left(P_{\mathrm{r}}-P_{\mathrm{r}}^{\prime}\right)$.

We assume that the real and assumed postions (coordinates) of the reference source are coincident, as expected in our case,
$P_{\mathrm{r}}-P_{\mathrm{r}}^{\prime}=0$. Then the observational position offset $P_{\mathrm{s}}^{\prime \prime}$ reflects the offset between the real and nominal coordinates of the target:

$P_{\mathrm{s}}^{\prime \prime}=P_{\mathrm{s}}-P_{\mathrm{s}}^{\prime}$.

In our observations, we have confirmed that the real and given coordinates of the reference source are coincident within the errors observing two reference sources (see below). In this way, we obtain maps including very accurate positions with respect to the reference source, and, therefore, very accurate astrometry measurements.

The $\mathrm{SiO}$ masers around $\mathrm{R}$ Aqr (Hipparcos coordinates: $\left.\alpha_{\mathrm{J} 2000}=23 \mathrm{~h} 43 \mathrm{~m} 49.4616 \mathrm{~s}, \delta_{\mathrm{J} 2000}=-15^{\circ} 17^{\prime} 04^{\prime \prime} .202\right)$ and the extragalactic source $\mathrm{J} 2348-1631\left(\alpha_{\mathrm{J} 2000}=23 \mathrm{~h} 48 \mathrm{~m} 02.608532 \mathrm{~s}\right.$, $\delta_{\mathrm{J} 2000}=-16^{\circ} 31^{\prime} 12^{\prime \prime}$.02226) were observed simultaneously using this system. Each observation lasted $\sim 7 \mathrm{~h}$, including scans of the targets and a calibrator (3C 454.3). The 16 IF channels of $16 \mathrm{MHz}$ bandwidth were recorded onto magnetic tapes at a rate of $1024 \mathrm{Mbps}$, of which two IF channels were assigned to the $v=1 J=1-0$ and $v=2 J=1-0 \mathrm{SiO}$ maser transitions toward R Aqr, and the other 14 IF channels were assigned to the position reference source J2348-1631.

A typical size of the synthesized beam was $0.8 \times$ 0.5 milliarcsec (mas) with PA $\sim-20^{\circ}$. The velocity resolution of the $\mathrm{SiO}$ maser transitions in three epochs, Dec. 2004, Dec. 2005, and August 2006, is $0.22 \mathrm{~km} \mathrm{~s}^{-1}$, and the velocity resolution in the other epochs is $0.11 \mathrm{~km} \mathrm{~s}^{-1}$.

The data reduction and the identification of maser spots were made using the NRAO AIPS packages. We followed three steps to obtain absolute coordinates of all maser spots. First of all, visibility amplitude calibration was made using the data of system noise temperatures independently measured in the two beams. Antenna gains were also calibrated using the tables obtained from previous antenna performance measurements. The bandpass amplitude characteristics were also measured with scans of continuum sources observed every $80 \mathrm{~min}$ and applied to the amplitude calibration. This calibration process was also followed independently for the individual beams.

The correction table of the instrumental phase difference described above was also applied. Because the a priori delay model applied in the correlation processing was not accurate enough to obtain precise astrometry, we calibrated the visibility phase using the most accurate delay model, based on the recent achievements of geodynamics. In this model, we calibrated the fluctuation of the visibility phase caused by Earth's atmosphere based on GPS measurements of the atmospheric zenith delay due to the tropospheric water vapor, see more details in Honma et al. (2008b). Then, fringe fitting was performed using the AIPS task FRING on the reference source, and the solutions were applied to the target source. This way, we obtained the positions of some simple and bright maser spots, identified by the closure phases, relative to the J2000 coordinate system using the AIPS task JMFIT.

Next, an interactive self-calibration and imaging procedures was performed to map a single strong reference spectral feature in each transition. Then we obtained CLEAN image cubes of the individual maser transitions. A CLEAN window was set to the whole image region. This setting automatically finds in the CLEAN iterations the highest brightness peaks above $800 \mathrm{mJy} /$ beam (approximately equivalent to a signal-to-noise ratio of at least a factor 5 and to a ratio between the strong and the weakest features of about 20). We only selected maser spots identified in three consecutive velocity channels and with positions overlapping within the beam size. We note that, in our case, the $\mathrm{SiO}$ maser spots around $\mathrm{R}$ Aqr are so strong that the 
Table 1. Summary of VERA observations in R Aqr. We give the relative positions of the central star with respect to the J2000 Hipparcos coordinates (and their uncertainties at $2 \sigma$-level).

\begin{tabular}{|c|c|c|c|c|c|c|}
\hline \multirow[t]{2}{*}{ Date } & \multicolumn{2}{|c|}{ Star's relative coordinates (mas) } & \multirow[b]{2}{*}{$v=1 J=1-0$} & \multicolumn{2}{|c|}{ Number of spots } & \multirow[t]{2}{*}{ Comments } \\
\hline & $\Delta(\mathrm{AR})$ & $\Delta(\mathrm{Dec})$ & & $v=2 J=1-0$ & coincident $v=1,2$ & \\
\hline 23 Dec. 2004 & $135.3(4.2)$ & $-133.1(1.4)$ & 7 & 41 & $1(1)$ & \\
\hline 27 Sep. 2005 & $161.4(0.5)$ & $-154.9(0.8)$ & 4 & 76 & $0(0)$ & \\
\hline 23 Nov. 2005 & $164.5(0.3)$ & $-160.7(0.5)$ & 79 & 77 & $3(11)$ & \\
\hline 24 Dec. 2005 & $166.4(0.3)$ & $-162.4(0.6)$ & 66 & 75 & $11(16)$ & \\
\hline 14 Feb. 2006 & $172.7(0.3)$ & $-165.2(0.5)$ & 116 & 157 & $0(1)$ & \\
\hline 04 Mar. 2006 & $175.1(0.3)$ & $-165.7(0.5)$ & 56 & 196 & $0(5)$ & \\
\hline 08 May 2006 & - & - & & & - & no absolute astrometry \\
\hline 11 Jul. 2006 & - & - & & & - & no absolute astrometry \\
\hline 26 Jul. 2006 & - & - & & & - & no absolute astrometry \\
\hline 30 Aug. 2006 & $195.7(1.0)$ & $-181.5(0.8)$ & 24 & 31 & $0(0)$ & \\
\hline 13 Oct. 2006 & $196.6(2.4)$ & $-188.4(1.0)$ & 41 & 73 & $4(6)$ & poor fitting \\
\hline
\end{tabular}

Notes. The spot coincidences have been estimated for spatial distances less than $1 / 3$ (and than 2/3) of the beam.

closure phases of each observation reflect the actual structure and distribution of the maser components. We determined maser spot distributions in our observations that yield accurate fittings of the clean model to the visibility phases (to better than $10^{\circ}$ ). However, the limited VERA's $(u, v)$ plane coverage may introduce artifacts around a bright feature. Because these artifacts tend to always appear in the same relative positions with respect to true bright maser spots, they could be identified after maser spot identification and were rejected. We have obtained positions of maser spots relative to the reference one using the AIPS task SAD. Finally, we compared the positions of identified maser spots of the phase-referenced images and the self-calibrated images, and calculated the offsets to be applied to the images to determine absolute coordinates of all maser spots.

We cannot exclude, in any case, a few of the weakest spikes appearing because of cleaning uncertainties. This would introduce some extra noise in the fitting of the circular maser distribution (Sect. 3), but would practically not affect our main conclusions. We checked in particular that our statistical results on the percentage of coincident spots of both maser lines (Sect. 4) do not significantly change if we only consider strong spikes, i.e. those showing a brightness greater than for instance 3 or $10 \mathrm{Jy} /$ beam in one of the lines.

To confirm the accuracy of the astrometric measurements and, in particular, of the relative positions derived for the spots of both transitions, we performed some observations in the two IF channels used for $\mathrm{SiO}$ masers of the extragalactic source $\mathrm{J} 2345-1555\left(\alpha_{\mathrm{J} 2000}=23 \mathrm{~h} 45 \mathrm{~m} 12.462326 \mathrm{~s}, \delta_{\mathrm{J} 2000}=\right.$ $-15^{\circ} 55^{\prime} 07^{\prime \prime} .83413$ ), which can be observed simultaneously with our reference source $\mathbf{J} 2348-1631$. The position difference for both channels was found to be very small, 0.04 mas in RA and 0.07 mas in declination. The relative positions are found, therefore, to be accurate within $\sim 1 / 10$ of the beam size.

We present here VERA observations of $\mathrm{SiO}$ masers at $\lambda=$ $7 \mathrm{~mm}$ (both $v=1$ and 2, $J=1-0$ lines) in R Aqr, performed between December 2004 and October 2006. Maps were obtained for a total of 11 epochs, in 8 of which we were able to obtain absolute astrometric measurements. More recent observations are still under analysis. Preliminary maps and analysis from the two first epochs have already been reported (Kamohara et al. 2008). A summary of the observations is given in Table 1, together with some results obtained from them (see below), and in Fig. 1, which shows the stellar light curve (kindly provided by the AAVSO) and the observation epochs. We recall that the $\mathrm{SiO}$ maxima in Mira-type variables usually appear at a phase

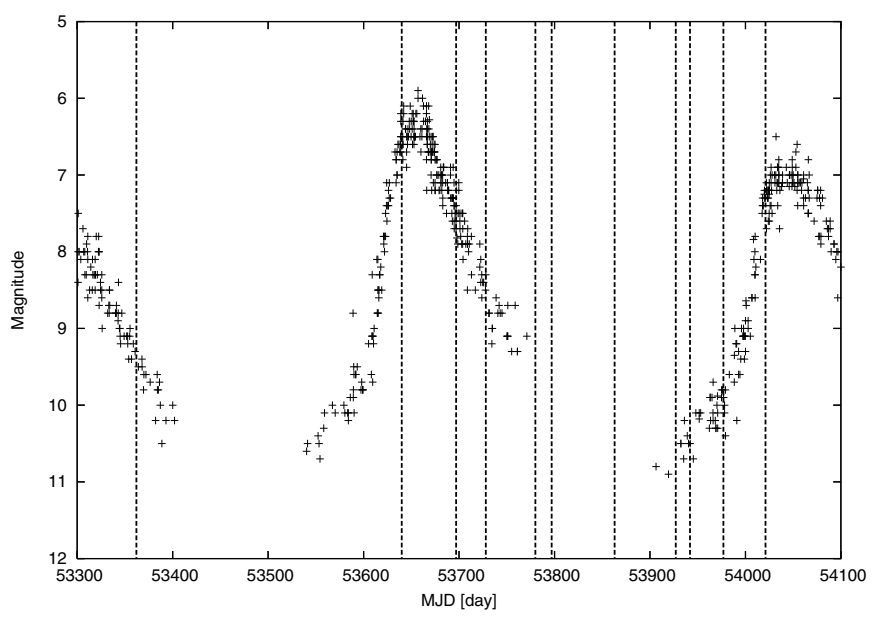

Fig. 1. Light curve in the visible of R Aqr, kindly provided by the AAVSO, and epochs of our observations (vertical lines; Julian Dates minus 2400000 days are given).

$\sim 0.1$ with respect to the optical maxima (Pardo et al. 2004). The resulting maps for the epochs in which absolute astrometry was performed are presented in Figs. 2 to 9. The relative coordinates are always given with respect to the J2000 Hipparcos coordinates $\left(\alpha_{\mathrm{J} 2000}=23 \mathrm{~h} 43 \mathrm{~m} 49.4616 \mathrm{~s}, \delta_{\mathrm{J} 2000}=-15^{\circ} 17^{\prime} 04^{\prime \prime} \cdot 202\right)$. See maps for the other epochs and some complementary results in the Appendices.

\section{Measurements of the parallax and proper motions of R Aqr}

The method of estimating annual parallaxes from VERA maps has usually been based on astrometric measurements of the position of a given maser spot (e.g. Nakagawa et al. 2008). In our case, this procedure is less appropriate, 1) because the whole emitting ring clearly shows sizeable radial motions, see below, with a period and amplitude comparable to those of the parallax movements; and 2) because the lifetimes of the spots are too short to be followed during our long observing period; in fact, we see in Sect. 5.2 that the shape of the emitting region has changed significantly.

$\mathrm{SiO}$ maser emission from AGB stars is known to form a ring of spots around the star, at a few stellar radii (see e.g. Cotton et al. 2006; Reid \& Menten 2007, and references therein). 

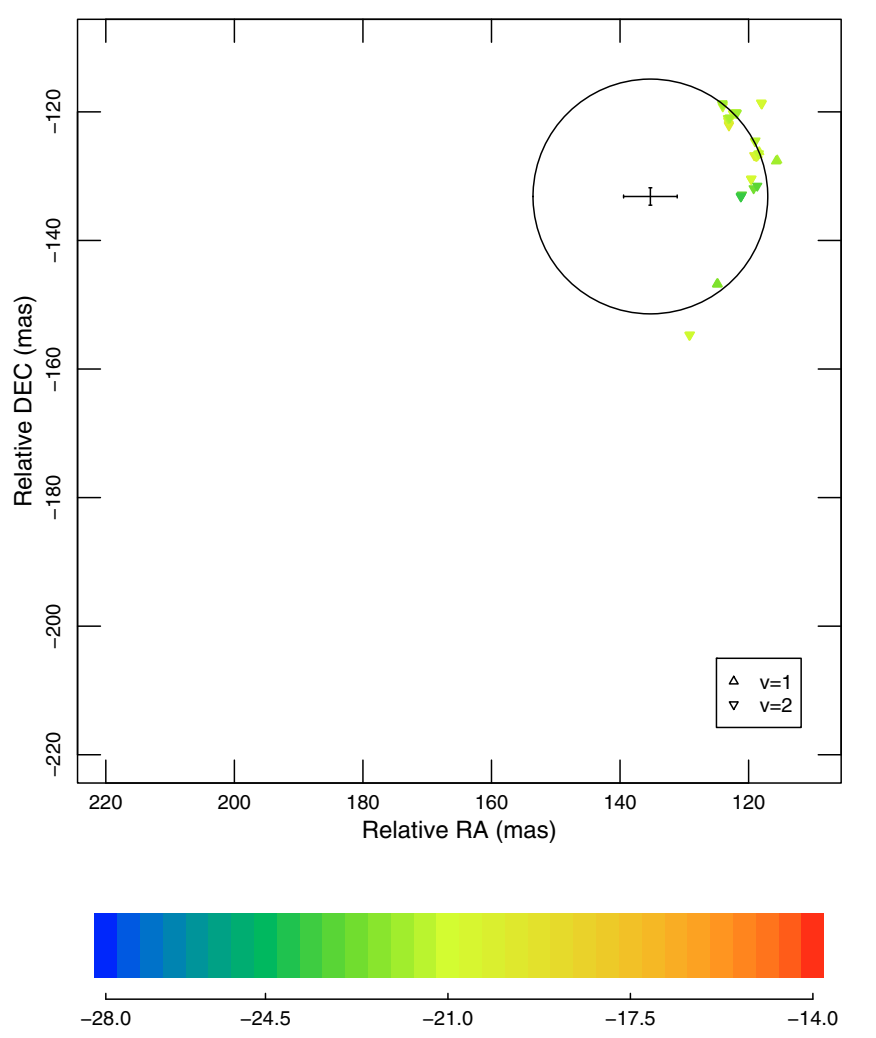

Fig. 2. Distribution of $\mathrm{SiO}$ masers around $\mathrm{R} A q r$, observed on 23 December 2004 (2004.98). The relative positions are given with respect to the J2000 Hipparcos coordinates. Triangles pointing upwards represent the distribution of the $v=1 J=1-0$ transition and those pointing downwards show the spots of the $v=2 J=1-0$ transition. The circle fitted to the maser spots is shown and the central cross marks its center and the uncertainty of the central position (at $2 \sigma$-level). The color scale represents the $L S R$ velocity.

Accordingly, we assumed that the star's center position is coincident with the center of a circle that is fitted to the maser spot distribution for each epoch. The uncertainties are given in terms of standard deviations assuming Gaussian dispersions for the different parameters. To improve the accuracy of the method, we fitted both the $v=1$ and $v=2 J=1-0$ maps. In Figs. 2 to 9, we show the circle resulting from this fitting, as well as its center and the uncertainty in the center coordinates. (We will always represent the centroid position limits by two times the formal uncertainties, $2 \times \sigma$, which gives more realistic boundaries to the true coordinates of the star's center.) We tested the goodness of the fit using a chi-square test. Except for the data on 13 October 2006 (which shows an anomalous distribution of difficult interpretation, see discussion in Sect. 5), the observed spot distributions are explained by the assumed ring-like distributions: the probability that the deviations from the model fitting comes from a random dispersion around a mean value is nearly $100 \%$.

The variations in the right ascension and declination offsets with respect to the J2000 Hipparcos coordinates of R Aqr $\left(\alpha_{\mathrm{J} 2000}=23 \mathrm{~h} 43 \mathrm{~m} 49.4616 \mathrm{~s}, \delta_{\mathrm{J} 2000}=-15^{\circ} 17^{\prime} 04^{\prime \prime} .202\right)$ shows the proper movement of the star from December 2004 to August 2006. The positions of the emission centroid calculated in this way are given in Table 1, except for the epochs in which no absolute coordinates were obtained. We show such displacements in Fig. 10, where the contribution from the annual parallax is evident.
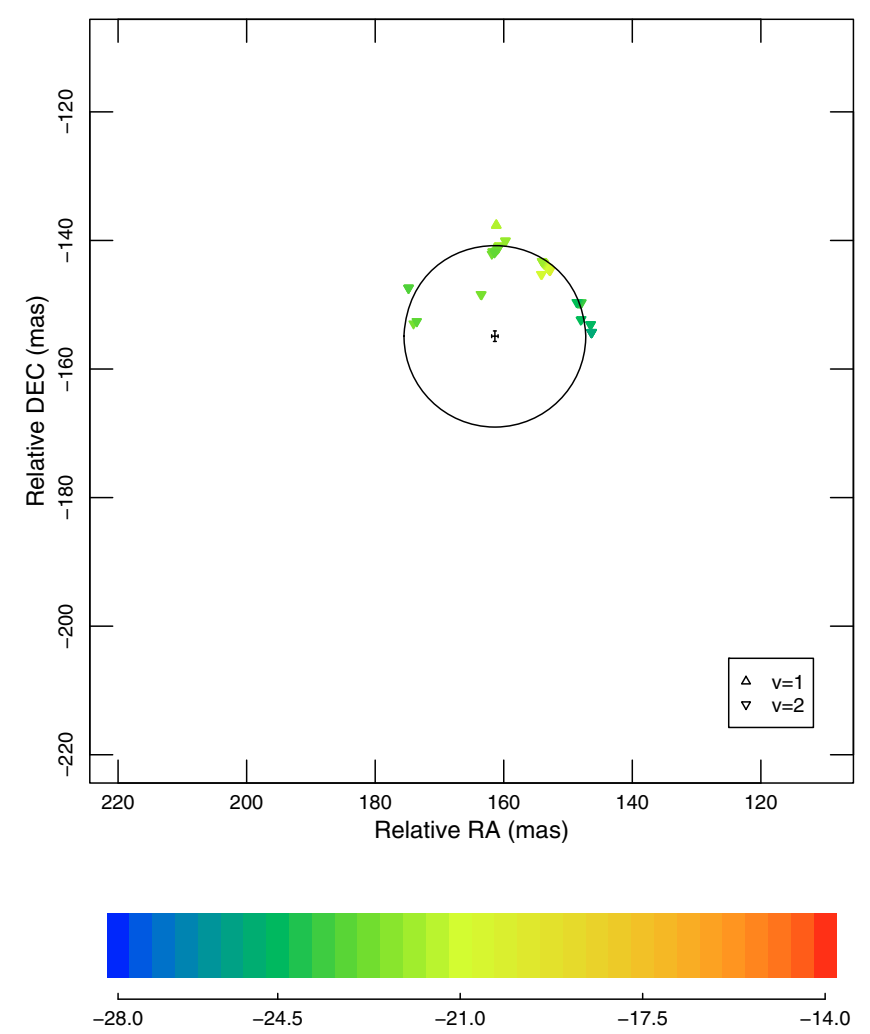

Fig. 3. Distribution of $\mathrm{SiO}$ masers around R Aqr on 27 September 2005. Same symbols and representation of the fitting results as in Fig. 2.

The parallax was estimated by fitting both RA and declination offsets simultaneously. In our method, we assume that proper motions, after the parallax is subtracted, are linear. Therefore, they include the linear component of the displacement of the red giant around the center of gravity of the double system. This contribution (at the distance of the source and for the probably low mass of the companion) is in any case expected to be minor. We find a value of $\pi=4.7 \pm 0.8$ mas, yielding a distance to R Aqr $D=214_{-32}^{+45} \mathrm{pc}$. Our measurement is significantly more accurate than the Hipparcos parallax $(5.07 \pm 3.15$ mas $)$, though both results are compatible. We therefore find that R Aqr is nearly $10 \%$ farther than what was previously believed. We show the residuals from the best-fit curve in Figs. 11 and 12.

Subtracting the parallax movements, we have estimated linear proper motions in right ascension and declination. We thus obtain $\mu_{\mathrm{RA}}=32.2 \pm 0.8 \mathrm{mas}^{\mathrm{yr}}{ }^{-1}$ and $\mu_{\mathrm{dec}}=-29.5 \pm$

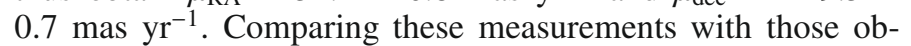
tained by Hipparcos $\left(\mu_{\mathrm{RA}}=32.98 \pm 1.46, \mu_{\mathrm{dec}}=-32.61 \pm\right.$ 1.13 ), one finds that the proper motion in RA is compatible within the errors, while the motion in declination is slightly different. The Hipparcos position (epoch 1991.25) is also compatible with our measurements of coordinates and proper motion, within the uncertainties and taking the error propagation along time into account, see more details in Kamohara et al. (2008). Although the orbital parameters of the binary system are still controversial, we can expect the time of apastron to be around 2001, adopting the oribital parameters estimated by Gromadzki \& Mikolajewska (2009), who propose a time of periastron passage $T_{0}=1979.40 \pm 1.99 \mathrm{yr}$ and a period of the orbit $P_{\text {orb }}=$ $43.64 \pm 1.29 \mathrm{yr}$. The binary components of R Aqr were resolved by Hollis et al. (1997) in November 1996. These data revealed that the Mira-like variable was located in the south of the system and the hot dwarf star was located in the north. Therefore, 
R. Kamohara et al.: VERA observations of $\mathrm{SiO}$ maser emission from R Aquarii
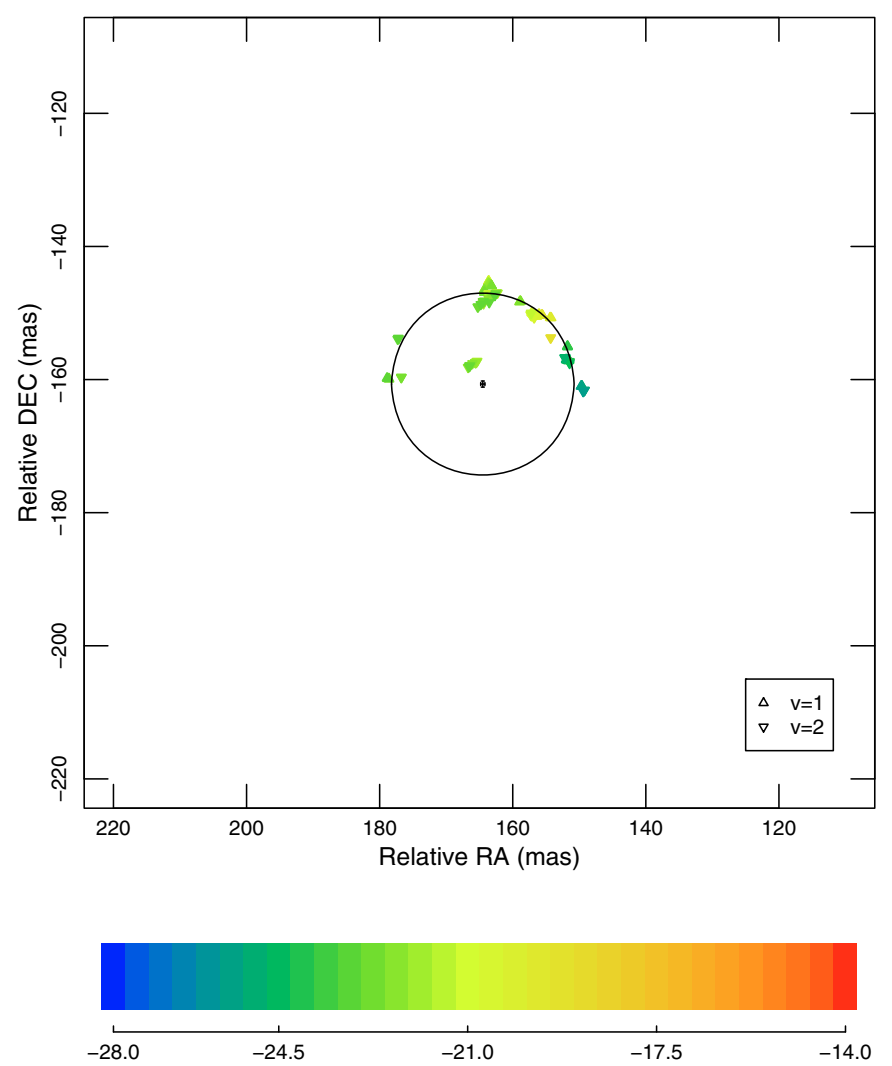

Fig. 4. Distribution of $\mathrm{SiO}$ masers around R Aqr on 23 November 2005. Same symbols and representation of the fitting results as in Fig. 2.

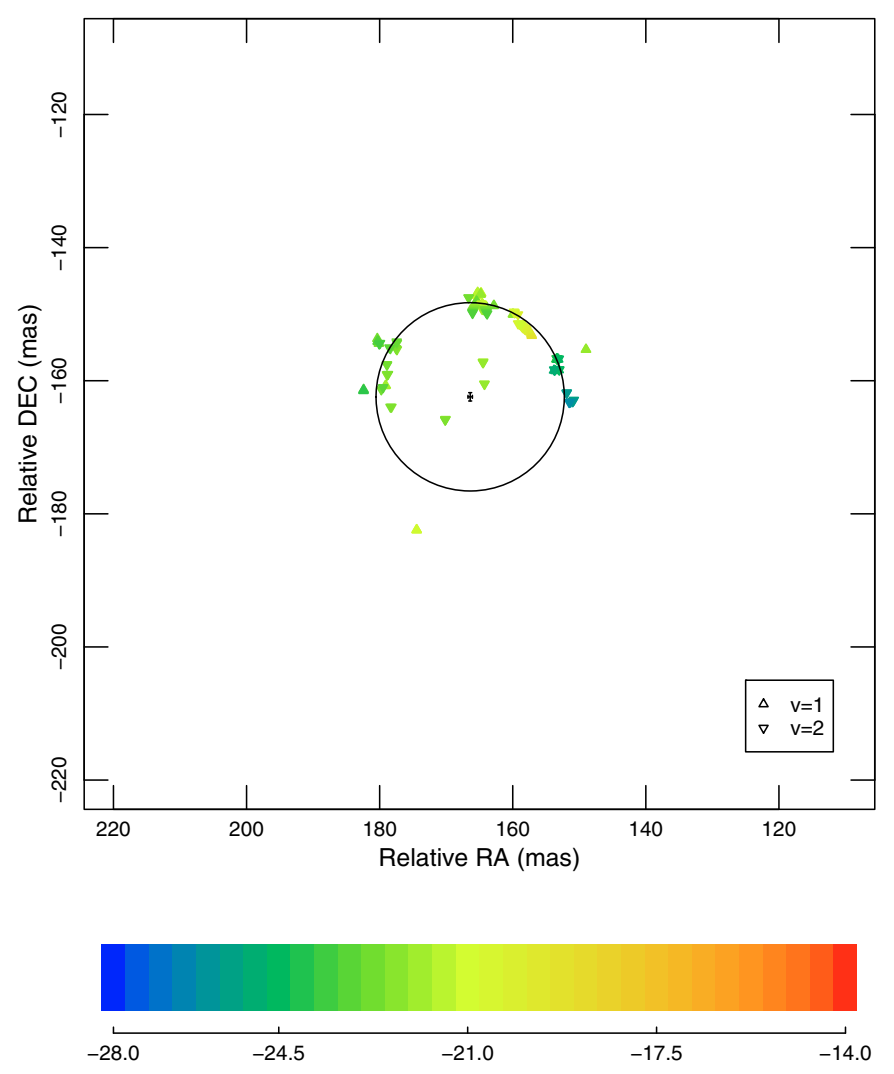

Fig. 5. Distribution of $\mathrm{SiO}$ masers around R Aqr on 24 December 2005. Same symbols and representation of the fitting results as in Fig. 2.
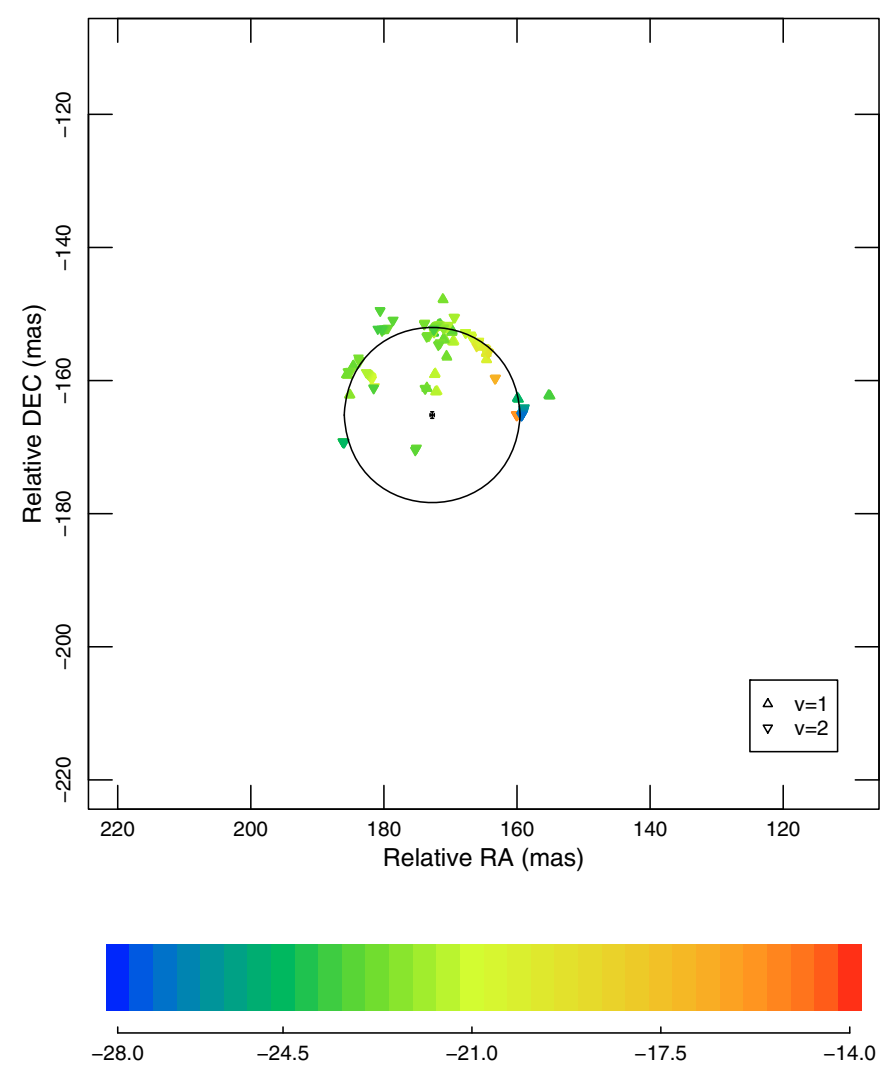

Fig. 6. Distribution of SiO masers around R Aqr on 14 February 2006. Same symbols and representation of the fitting results as in Fig. 2.

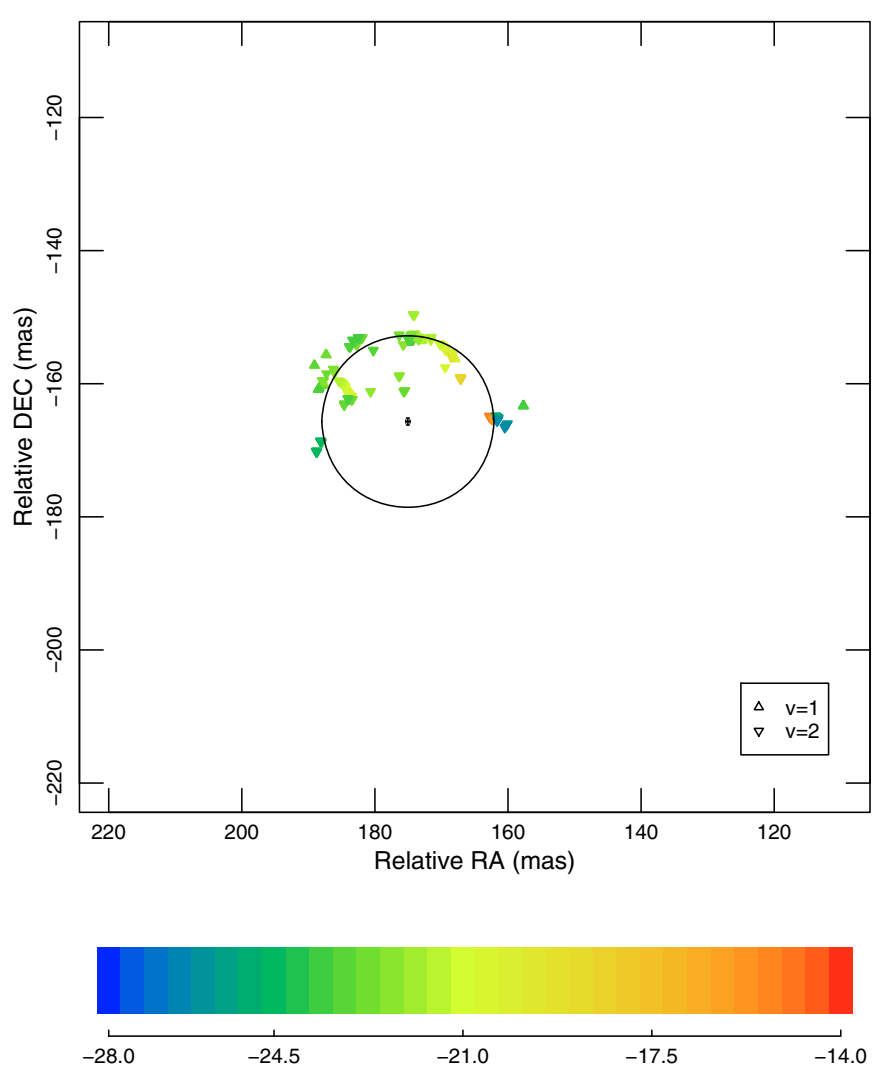

Fig. 7. Distribution of $\mathrm{SiO}$ masers around R Aqr on 4 March 2006. Same symbols and representation of the fitting results as in Fig. 2. 

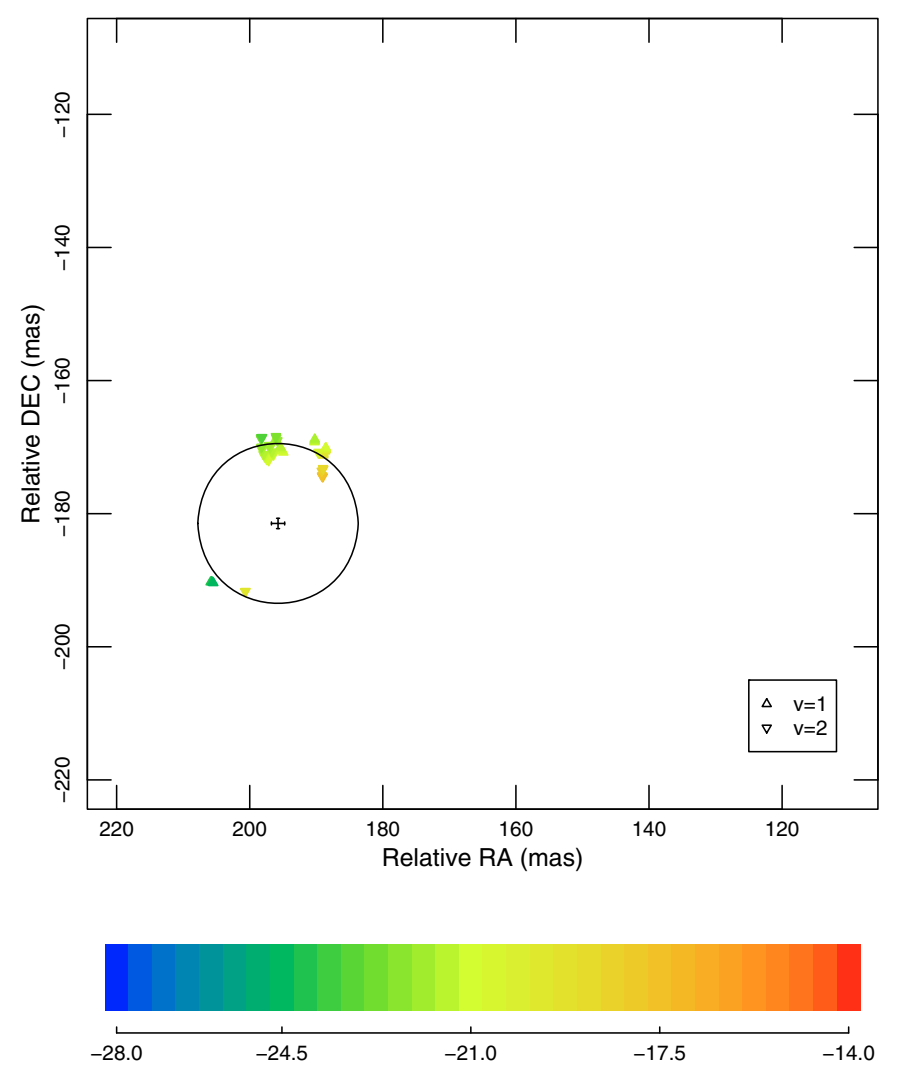

Fig. 8. Distribution of $\mathrm{SiO}$ masers around R Aqr on 30 August 2006. Same symbols and representation of the fitting results as in Fig. 2.

the sign of the orbital motions should have changed precisely around 2001, in a sense depending on the rest of the orbital elements. The difference found between the measurements of $\mu_{\mathrm{dec}}$ from Hipparcos data and ours could be related to this effect. However, we note that the difference between both measurements is scarcely significant; obviously, more observations are needed to draw conclusions on the orbital motion from VERA $\mathrm{SiO}$ maps.

Finally, we also estimated the variation with time of the circle radius fitted to the spot distribution, see results in Fig. 13. As we can see, there is a dominant infall kinematics in the whole emitting region. We discuss this result more in detail in Sect. 5.

\section{Relative positions of the $v=1$ and $v=2 J=1-0$ maser spots}

Figures 2 to 9 show the spatial distribution of the $\mathrm{SiO}$ maser spots, as well as their $L S R$ velocities (see also Appendix B), derived from our maps from December 2004 to October 2006, for both observed transitions ( $v=1 J=1-0$ and $v=2 J=1-0)$. Our observations of R Aqr follow the general trends of standard AGB stars, mentioned in Sect. 1.

\section{1. $v=1$ and $v=2 J=1-0$ masers: relative spatial and kinematical distributions}

Thanks to the very high astrometric accuracy of VERA, the absolute coordinates of the $v=1$ and $v=2$ spots in our maps have been very accurately measured. In particular, we have properly estimated the percentage of coincidences between spots of both transitions. We considered two spots of the two transitions to be coincident when they are separated by less than $1 / 3$ of the beam
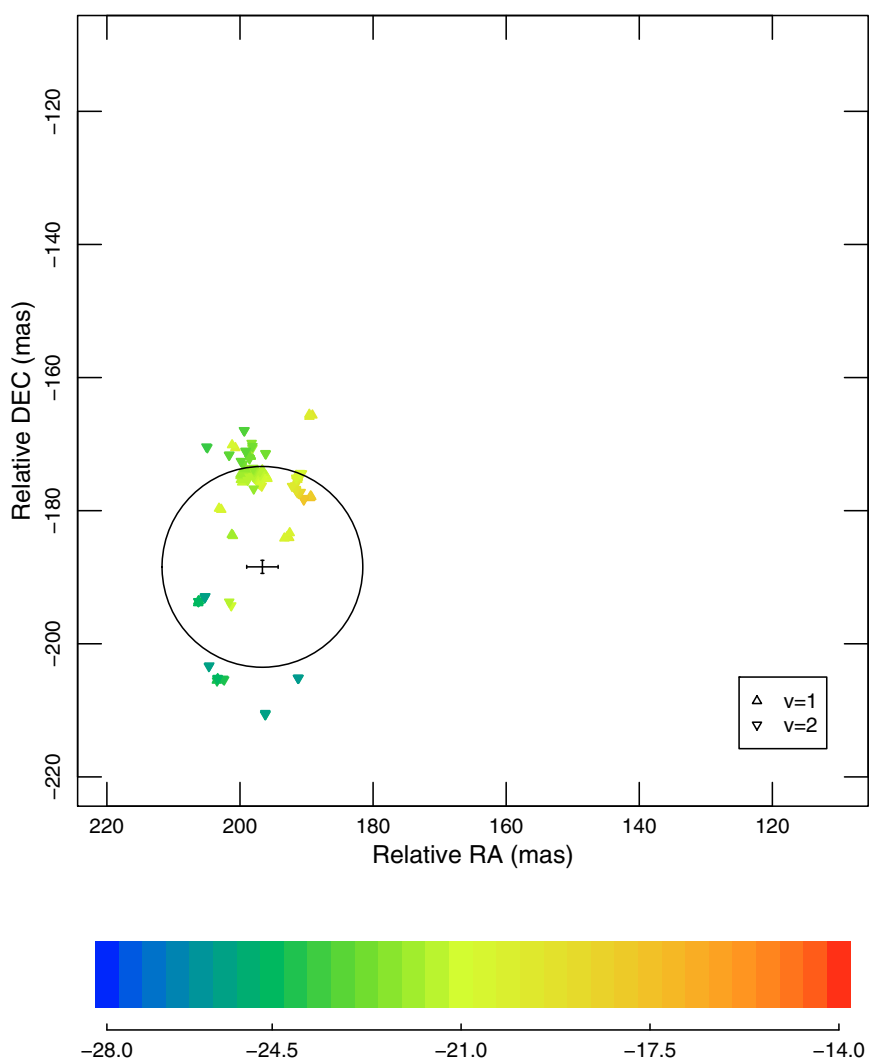

Fig. 9. Distribution of SiO masers around R Aqr on 13 October 2006. Same symbols and representation of the fitting results as in Fig. 2.

size (typically $0.8 \times 0.5$ mas) and are emitting in the same velocity within the spectral resolution of the observation (between 0.1 and $0.2 \mathrm{~km} \mathrm{~s}^{-1}$ ). The results are very similar if we take as condition being closer than $1 / 2$ or $2 / 3$ of the beam size. The numbers of spots identified for each transition and of coincidences are given in Table 1, for all epochs for which the comparison was possible (i.e. with good absolute astrometry), together with other results from our observations. We recall that the high quality of the VERA astrometric measurements allows this accurate comparison and insures that these coincidence statistics are reliable.

As we see in Table 1, December 2005 is the epoch showing the largest number of coincidences: $\sim 15 \%$ of the spots appear simultaneously emitting in $v=1$ and $v=2 J=1-0$ lines. In the other epochs, the coincidences are still rarer. In about one half of the observed epochs, there is no coincidence at all; the case of the observations performed in February and March 2006 is remarkable, because of the many spots identified in our maps.

Therefore, we accurately and quantitatively confirm the conclusion by Desmurs et al. (2000): both maser lines come from similar circumstellar regions, but their spots are only coincident in a small fraction of the cases. Sometimes (see e.g. epoch Dec. 2005, LSR intervals -22:-21, -23:-22, and $-24:-23 \mathrm{~km} \mathrm{~s}^{-1}$ ), the spots of both lines are very close but not coincident, separated by 1-2 mas, forming spot clusters and giving the impression of being grouped within a gas condensation. Rioja et al. (2008) show maps of R LMi in both transition with accurate relative astrometry. In one of their observation epochs (April 2007), the only one with distributions that are complex enough to allow an analysis of this kind, the $v=1$ and $v=2 J=$ $1-0$ spots were also grouped in clusters but separated by a small angular distance. 
R. Kamohara et al.: VERA observations of $\mathrm{SiO}$ maser emission from R Aquarii

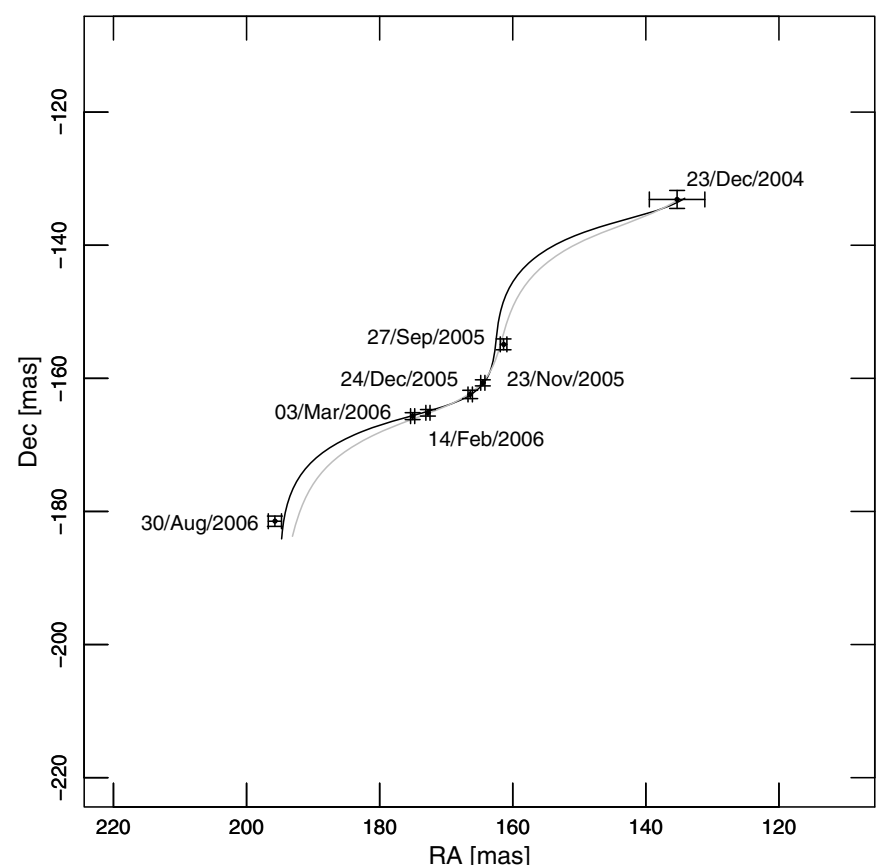

Fig. 10. Relative position of the $\mathrm{SiO}$ maser centroid measured in $\mathrm{R}$ Aqr, with respect with the Hipparcos coordinates (epoch J2000.0). We have also drawn the position uncertainties and our best fitting of the annual parallax and linear proper motions (Sect. 3). The gray line represents the parallax fitting obtained if the last epoch, 30/Aug./2006, is not taken into acount; see Appendix B for more details.

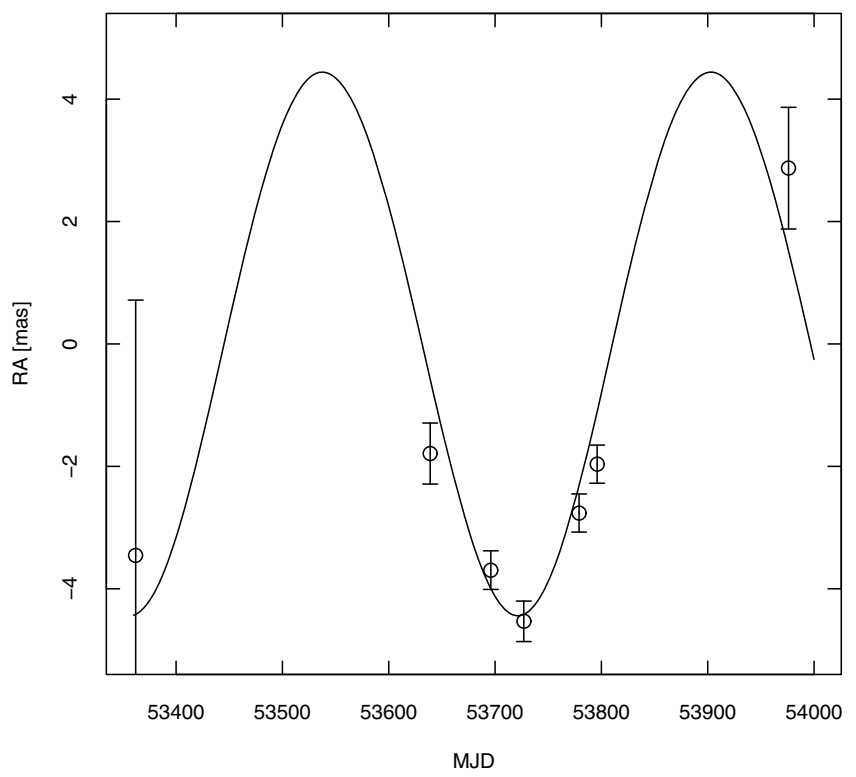

Fig. 11. Relative right ascension of the $\mathrm{SiO}$ maser centroid measured in R Aqr, after subtracting the linear proper motion, and the parallax movement fitted to the data.

In general and more noticeably when relatively complete rings of spots are detected, the $v=2 J=1-0$ spots tend to be slightly closer to the star than the $v=1$ ones, also confirming previous results. In some cases (December 2004, July 2006, August 2006, October 2006), the spots were mostly aligned in the west or east parts of the envelope for both $v=1$ and $v=2$ lines. In all epochs but one, the $v=2 J=1-0$ maser presents significantly more spots than the $v=1 J=1-0$ one, but

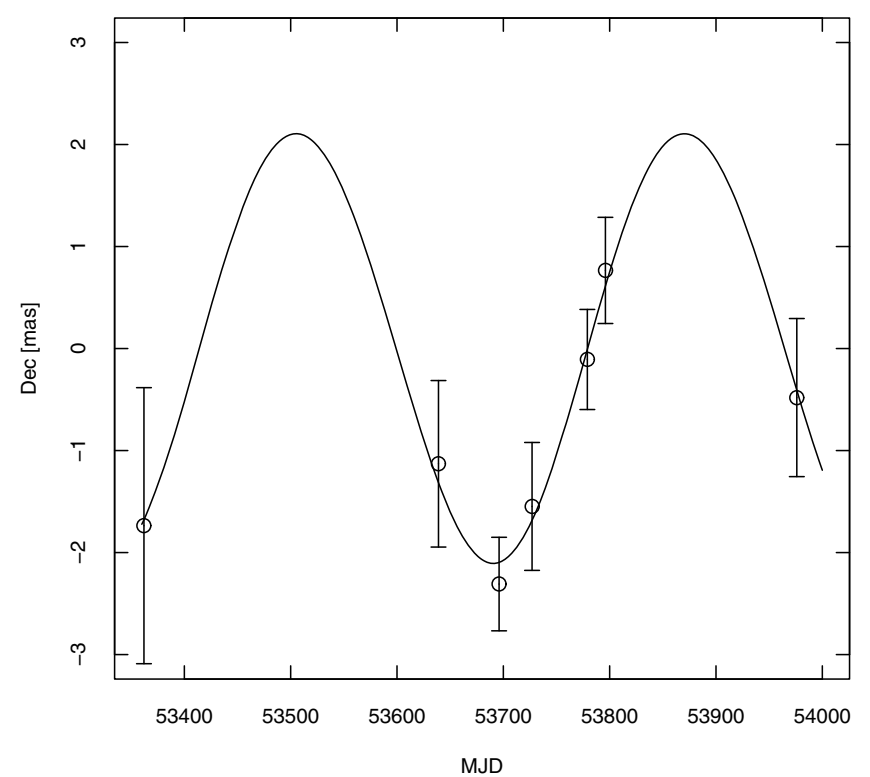

Fig. 12. Relative declination of the $\mathrm{SiO}$ maser centroid measured in $\mathrm{R}$ Aqr, after subtracting the linear proper motion, and the parallax movement fitted to the data.

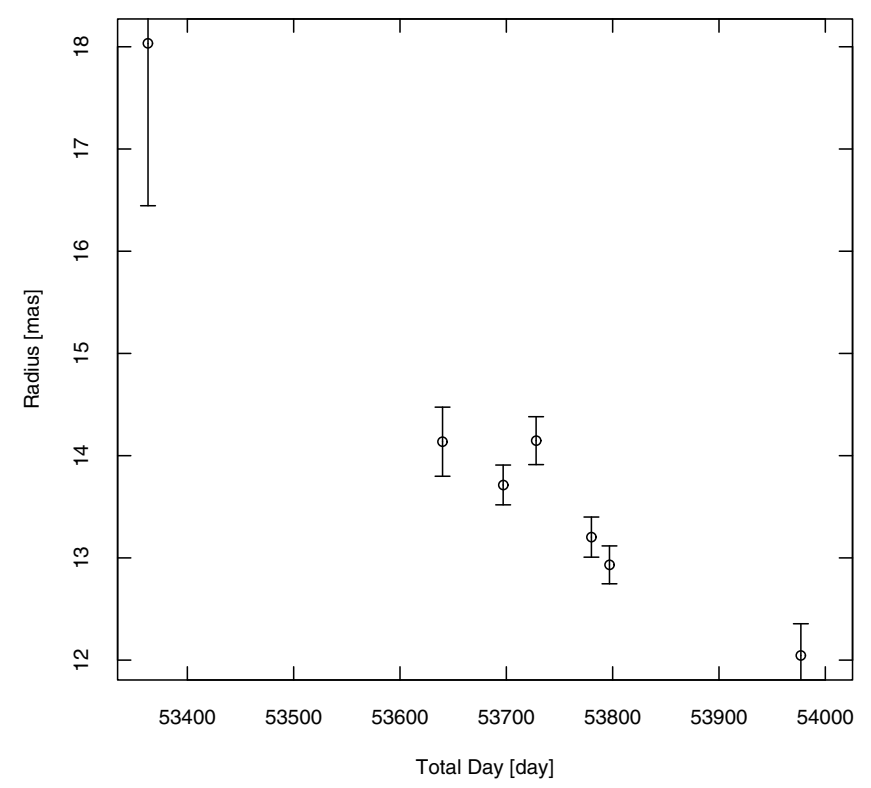

Fig. 13. Values of the radii of the emitting region as a function of time (Julian Date minus 2400000 ). Note that 15 mas is equivalent to $4.8 \times$ $10^{13} \mathrm{~cm}$ at $214 \mathrm{pc}$.

both lines occupy similar regions around the star. See discussion of the variation of the overall distribution in Sect. 5.2.

\subsection{Comparison with theoretical model predictions}

Theoretical models explain most observed properties of $\mathrm{SiO}$ maser emission, including the presence of rings of spots in many cases (Sect. 1). We have also mentioned that the relative distributions of the $v=1$ and $v=2 J=1-0$ maser spots, which occupy similar regions but are rarely coincident, is more controversial. In Sect. 4.1, we observationally studied the degree of coincidence of the spots emitting in these transitions from very accurate astrometric measurements, for the first time, obtaining a quantitative estimate of the percentage of coincidences. 
In the standard versions of both radiative and collisional pumping schemes (e.g. Bujarrabal \& Nguyen-Q-Rieu 1981; Lockett \& Elitzur 1992), the inversion of the $v=1$ and $v=2$ rotational transitions appear under quite different conditions: the $v=1$ state is at an energy of $1800 \mathrm{~K}$ over the ground level, while the $v=2$ one is at $3600 \mathrm{~K}$, so different (always high) excitation would be required. Therefore, the conditions under which both masers can coexist are rare in both models. In the radiative model, the coincidence is somewhat less probable than for the collisional pumping, because high optical depths in the $v=2 \rightarrow v=1$ rovibrational transitions (needed to invert the $v=2$ transitions) tend to quench the $v=1$ masers and such radiative phenomenon is less noticeable when collisional rates are high. This difference has led to the long-lasting discussion of the consequences on theoretical models of the possible systematic coincidence of $v=1$ and $v=2 J=1-0$ spots. Both pumping mechanisms predict that masers in rotational transitions within the same vibrational state $(J=1-0, J=2-1$, etc.) must be strongly coupled and should appear in practically the same points.

The discussion of this topic has dramatically changed from when the first comparisons between $v=1 J=1-0$ and $J=$ 2-1 maser distributions were performed (Soria-Ruiz et al. 2004, $2005,2007)$. The $v=1 J=2-1$ maser spots systematically occupy a ring with a significantly larger radius than that of $v=1$ $J=1-0$, and both spot distributions are completely unrelated. (We recall that the distributions of $v=1,2 J=1-0$ masers are always similar.) The $v=2 J=2-1$ transition is known to be anomalously weak, and practically no VLBI map of its distribution exists (e.g. Olofsson et al. 1981, see further details below).

Soria-Ruiz et al. (2004) interpret these results invoking line overlap between the rovibrational transitions $v=1, J=0-$ $v=2, J=1$ of $\mathrm{SiO}$, and $v_{2}=0,12_{7,5}-v_{2}=1,11_{6,6}$ of $\mathrm{H}_{2} \mathrm{O}$; see also Olofsson et al. (1981) and Bujarrabal et al. (1996). These infrared transitions have very similar frequencies and can radiatively interact. Soria-Ruiz et al. (2004) use a radiative pumping scheme for $\mathrm{SiO}$ masers, coupled with a model for $\mathrm{H}_{2} \mathrm{O}$ excitation, which allowed those authors to study the effects of the overlapping line pair onto $\mathrm{SiO}$ masers. (Since line overlap is a radiative phenomenon, its effects are always more noticeable when the population transfer between levels mostly occur by means of radiative transitions, rather than when collisions dominate.) Their calculations confirm that this line overlap relatively overpopulates the $v=1 J=0$ level and depopulates the $v=2 J=1$ level, therefore reinforcing the $v=1,2 J=1-0$ masers. The quenching of the (otherwise intense) $v=2 J=2-1$ maser is also produced, in agreement with observations. A very important consequence is that, according to Soria-Ruiz et al. (2004), the overlap introduces a strong coupling between $v=1,2 J=1-0$ masers, which now are predicted to require very similar conditions to be intense, while the $v=1 J=2-1$ maser is less affected by this phenomenon and its masing conditions become significantly different from those for the other two lines. Nevertheless, the spatial coincidence of the $v=1,2 J=1-0$ masers is not predicted to be exact, probably because the $\mathrm{SiO}$ vibrational excitation is radiative in this model, and some difference in the brightness distribution persists. We think that we cannot understand the high-resolution maps of $\mathrm{SiO}$ masers without taking those theoretical considerations on the effects of line overlap into account.

Our VERA absolute-coordinates maps very accurately confirm previous results on the $v=1,2 J=1-0$ maser distributions. The spots of both transitions are placed in similar regions, but are rarely coincident and the $v=2 J=1-0$ spot ring is slightly closer to the star. All the relevant observational data agree (at least qualitatively) with the results of radiative pumping models including line overlap, as proposed by Soria-Ruiz et al. (2004). The $v=2 J=1-0$ maser is predicted by the model to be at a slightly shorter distance than $v=1 J=1-0$, by about $5-10 \%$ of the radius, the $v=1 J=2-1$ should be clearly farther (and require quite different excitation conditions), and the $v=2$ $J=2-1$ maser should practically be not excited.

However, we still lack specific theoretical studies of the meaning of the often observed grouping of spots of both $v=1$ and $2 J=1-0$ lines, and of whether the observed structure correspond to clumps in the inner circumstellar shell. Such studies are necessary for deducing conclusions on the inner shell structure from the existing very accurate $\mathrm{SiO}$ maser maps. Under this scenario, we should also investigate the theoretical predictions for the probability of detecting, for both $v=1$ and $v=2 J=1-0$, coincident spots with very high brightness, i.e. detectable in VLBI. The existing predictions mainly concern the overall shape of the maser region, but it is not clear in which way condensations may host maser spots of several maser transitions. It is in particular not clear if the lines of sight yielding a very high brightness (and involving very saturated maser emission) of both $v=1$ and $v=2 J=1-0$ masers can be coincident, or if some kind of maser competition (under high-saturation conditions) in fact prevents such a coincidence. Further theoretical studies in this direction, and including line overlap efects, are obviously required.

\section{Overall distribution of SiO maser emission in R Aqr and its variation with time}

\subsection{Velocity distribution}

Previous observations of $\mathrm{SiO}$ emission from $\mathrm{R}$ Aqr have sometimes presented a regular pattern in velocity, which has been attributed by certain authors to rotation in the inner circumstellar layers around the Mira-like variable of the binary system. We present in Table 2 a summary of the main properties of the $\mathrm{SiO}$ maser distribution in R Aqr, observed from June 1995 to October 2006 by several authors. A total of 23 observations, 20 of which are VLBI high-resolution maps, are discussed, including our data, a total of eleven epochs.

Hollis et al. (2000) obtained low-resolution maps, from connected interferometry, between November 1996 and February 2000. They identified a velocity gradient that was interpreted as a possible rotation of the shell around an axis oriented at PA $\sim 45^{\circ}$. Hollis et al. (2001) performed further $\mathrm{SiO}$ observations in December 2000, this time using the VLBA and attaining very high resolution. They claim that Keplerian rotation was detected, but the axis was almost exactly perpendicular to the one found before, $\mathrm{PA} \sim-40^{\circ}$.

As we can see in our maps (Figs. 2 to 9 and B.1 to B.3), the velocities of the different spots mostly present erratic distributions. This applies to all epochs, i.e. from 2004 to 2006 observations (including the data by Ragland et al. 2008, obtained in September 2006), but is clearer in 2005 and early 2006 data, since the spots occupy a wider region. In practically all cases, the velocity distribution is not compatible with rotation (Table 2).

In our data obtained in 2005 (September, November, and December) and most of our data in 2006 (February, March, May), the spot ring around the star is more complete, occupying the northern part of the shell. The extreme blue- and red-shifted emission comes from the west part of the shell. The spots in the east have velocities between -22 and $-24 \mathrm{~km} \mathrm{~s}^{-1}$, and there is a centered component at velocities between -21 and $-23 \mathrm{~km} \mathrm{~s}^{-1}$. 
Table 2. Summary of all relevant maps of $\mathrm{SiO}$ maser emission obtained in $\mathrm{R}$ Aqr.

\begin{tabular}{|c|c|c|c|c|c|}
\hline Date & $\begin{array}{c}\text { Type of } \\
\text { observation }\end{array}$ & $\begin{array}{c}\text { Axis of symmetry/elongation } \\
\text { PA }\end{array}$ & $\begin{array}{c}\text { Rotation axis } \\
\text { PA }\end{array}$ & $\begin{array}{l}\text { Dominant } \\
\text { half? }\end{array}$ & Reference \\
\hline June 1995 & VLBI & $-20^{\circ}$ & $?$ & west & Boboltz et al. (1997) \\
\hline Dec. 1995 & VLBI & $-20^{\circ}$ & ? & & Boboltz et al. (1997) \\
\hline Feb. 1996 & VLBI & $-20^{\circ}$ & $?$ & & Boboltz et al. (1997) \\
\hline Apr. 1996 & VLBI & $-20^{\circ}$ & $?$ & & Boboltz et al. (1997) \\
\hline Nov. 1996 & conn. interf. & $\pm 45^{\circ} ?$ & $+45^{\circ}$ & & Hollis et al. (2000) \\
\hline May 1998 & conn. interf. & $\pm 45^{\circ} ?$ & $+45^{\circ}$ & & Hollis et al. (2000) \\
\hline Dec. 1999-Feb. 2000 & conn. interf. & $\pm 45^{\circ} ?$ & $+45^{\circ}$ & & Hollis et al. (2000) \\
\hline Dec. 2000 & VLBI & $-40^{\circ}$ & $-40^{\circ}$ & & Hollis et al. (2001) \\
\hline Jan. 2001 & VLBI & $-10^{\circ}$ & $-10^{\circ}$ & & Cotton et al. (2004) \\
\hline Apr. 2001 & VLBI & $-10^{\circ} ?$ & $-10^{\circ}$ & & Cotton et al. (2004) \\
\hline Sep. 2004 & VLBI & $0^{\circ}$ & no & west & Cotton et al. (2006) \\
\hline Dec. 2004 & VLBI & $-10^{\circ}$ & no & southwest & this paper \\
\hline Sep. 2005 & VLBI & $0^{\circ}$ & no & north & this paper \\
\hline Nov. 2005 & VLBI & $0^{\circ}$ & no & north & this paper \\
\hline Dec. 2005 & VLBI & $0^{\circ}$ & no & north & this paper \\
\hline Feb. 2006 & VLBI & $0^{\circ}$ & no & north & this paper \\
\hline Mar. 2006 & VLBI & $0^{\circ}$ & no & north & this paper \\
\hline May 2006 & VLBI & $?$ & $?$ & north? & this paper (no astrometry) \\
\hline Jul. 2006 & VLBI & $?$ & $?$ & northeast? & this paper (no astrometry) \\
\hline Jul. 2006 & VLBI & $?$ & $?$ & northeast? & this paper (no astrometry) \\
\hline Aug. 2006 & VLBI & $-10^{\circ}$ & ? & northeast & this paper \\
\hline Sep. 2006 & VLBI & $-10^{\circ}$ & no & northeast & Ragland et al. (2008) \\
\hline Oct. 2006 & VLBI & $0^{\circ}$ & no & northeast? & this paper \\
\hline
\end{tabular}

At about $-23 \mathrm{~km} \mathrm{~s}^{-1}$ the emission occupies almost all the northern hemisphere. This distribution is completely different from given by any rotation velocity field.

In summary, some of the first performed maps of $\mathrm{SiO}$ emission from R Aqr are compatible with Keplerian rotation, but indicating significantly different rotation axes. Later data are, moreover, incompatible with the presence of rotation.

However, other velocity fields are likely to be present in R Aqr. Boboltz et al. (1997) do not publish the LSR velocity of emission features, but they calculate proper motions (in the plane of the sky) of the different spots. They find a systematic component that mostly corresponds to infall, with a velocity of $4.1 \pm 0.9 \mathrm{~km} \mathrm{~s}^{-1}$ (for the distance found by us, $214 \mathrm{pc}$, Sect. 3), which agrees with the expected velocities due to pulsation propagating in these circumstellar shells. Our data also suggest radial motions, see Sect. 3 and Fig. 13. We estimate an infall velocity $\sim 3.1 \pm 0.6 \mathrm{~km} \mathrm{~s}^{-1}$, reasonably compatible with the value obtained by Boboltz et al. (1997). These results on R Aqr agree with measurements in other objects. The best example is TX Cam, see Gonidakis et al. (2006), where the maser spots are clearly found to move radially.

\subsection{Spatial distribution}

Independently of the velocity pattern, the $\mathrm{SiO}$ emitting region in R Aqr sometimes presents a peculiar spatial distribution, showing in particular clear axes of symmetry or elongations. The $\mathrm{SiO}$ maser distribution in our first observations, December 2004, occupies a north-south elongated region, in the western part of the circumstellar shell (very similar to the structure found in September 2004 by Cotton et al. 2006). In our data from September 2005 to May 2006, the $\mathrm{SiO}$ maser region is the northern part of the shell, with a clear axis of symmetry at PA $\sim 0^{\circ}$. Finally, in our observations of August 2006, probably also in those of July 2006 and October 2006 (as well as in those by
Ragland et al., obtained in September 2006), the SiO emitting region is placed eastwards of the star and is elongated along the direction $\mathrm{PA} \sim-10^{\circ}$. We must keep in mind, in any case, that a significant amount of flux is filtered-out by our interferometric observations: we have typically recovered only about $40 \%$ of the total fluxes.

Therefore, most of the many $\mathrm{SiO}$ maps of $\mathrm{R}$ Aqr published to date present a symmetry axis with direction ranging from $\mathrm{PA}=0^{\circ}$ to $-30^{\circ}$, independent of the velocity field, and practically all the existing data are compatible with a structure of this kind (see also Boboltz et al. 1997; Hollis et al. 2000, 2001; Cotton et al. 2004). A summary of the published data is presented in Table 2. In some epochs (June 1995, September 2004, December 2004, and August to October 2006), the emission comes almost only from an elongated, sometimes quite straight rim.

$\mathrm{R}$ Aqr is a symbiotic binary system in strong interaction, therefore the inner circumstellar shells around the cool star, from which the $\mathrm{SiO}$ maser emission is expected to come, must also be strongly affected by the presence of the companion. This strong interaction is thought to be responsible, in particular, for the weak emission of $\mathrm{R}$ Aqr in $\mathrm{H}_{2} \mathrm{O}$ maser, $\mathrm{OH}$ maser, and CO thermal lines (Ivison et al. 1994, 1995; Groenewegen et al. 1999), which are expected to come from regions outer than the SiO-emitting shell. Moreover, the AGB stars in symbiotic systems present systematically higher mass-loss rates than isolated evolved stars (e.g. Mikołajewska 2007), which indicates that the gravitational interaction between both stars affects the mass ejection process or that accretion (basic in the symbiotic behavior) is fed by the inner circumstellar shells. Also by taking into account that the axes of the maser shell and of the binary orbit seem to be roughly parallel, we suggest that there is a relation between the remarkable axial symmetry of the $\mathrm{SiO}$ emitting region, confirmed in many maps obtained between 1995 and 2006, and the binary nature of this source. However, we do not think that the 
changes found in the shape of the $\mathrm{SiO}$ images (eastern or western rims, northern cap) can come from changes in the relative position of the companion, since the variation in the $\mathrm{SiO}$ emission occurs in much shorter times than the binary period, $44 \mathrm{yr}$ (Sect. 1).

\section{Conclusions}

We present VERA observations of $\mathrm{R}$ Aqr in $\mathrm{SiO}$ maser emission at $7 \mathrm{~mm}, v=1$ and $v=2 J=1-0$ transitions. Data of eleven epochs were obtained from December 2004 to October 2006, see Table 1 and Fig. 1. R Aqr is a well known symbiotic system including a Mira-type variable, around which the maser is expected to form, and a hot, compact companion. The VERA double-beam system allows the simultaneous observation of a continuum source, in general a quasar, and therefore provides very accurate measurements of absolute coordinates of the target. In eight of our observations, the system worked perfectly and we obtained accurate coordinates of the different spots, but we could only measure relative positions for the other three epochs. See our maps in Figs. 2 to 9 and in the Appendices.

We also reviewed the rest of the $\mathrm{SiO}$ maser maps published to date (Sect. 5). In total, $\mathrm{SiO}$ maps have been obtained in 23 epochs from 1995 to 2006, 20 of them VLBI high-resolution observations; see a summary in Table 2.

From our observation, we have been able to derive the parallax and linear proper motion of $\mathrm{R}$ Aqr. We find $\pi=4.7 \pm$ 0.8 mas, yielding a distance to R Aqr $D=214_{-32}^{+45} \mathrm{pc}$, significantly more accurate than the Hipparcos measurement $(\pi=5.1 \pm$ 3.2 mas). The linear proper motion values we find are $\mu_{\mathrm{RA}}=$

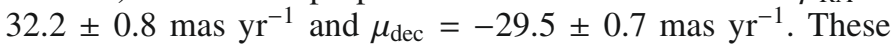
values are again more accurate than (and only slightly different from) the Hipparcos measurements $\left(\mu_{\mathrm{RA}}=32.98 \pm 1.46, \mu_{\mathrm{dec}}=\right.$ $-32.61 \pm 1.13)$. See discussion of the calculation method and uncertainties in Sect. 3. The non-linear components of the orbital motions within the binary system may partially contribute to the discrepancy in the proper motion measurements. However, due to the uncertainties discussed in Sects. 2 and 3, the existing data from $\mathrm{SiO}$ maps are still insufficient to improve the determination of the orbital parameters.

Thanks to the absolute coordinates derived from the VERA observations, we have been able to determine very accurately the relative positions of the $J=1-0 v=1$ and $v=2$ spots. We find that the distributions of both lines are very similar, the spots being often grouped defining clumps with a size of a few mas, but the spots of both transitions are rarely coincident. See our description of the spot coincidence analysis in Sect. 4.1, and a summary of the coincidence statistics in Table 1. Previous similar results have been proposed by several authors from standard VLBI experiments not including absolute coordinate measurements (e.g. Desmurs et al. 2000; Matsumoto et al. 2008). But, because of the lack of absolute astrometry, the comparison of the positions of both lines in those observations was difficult and the results were uncertain and controversial. See discussion of the implications of these results on the theoretical interpretation of $\mathrm{SiO}$ maser formation in Sect. 4.2. We conclude that radiative pumping models including line overlap effects (between two rovibrational components of respectively $\mathrm{H}_{2} \mathrm{O}$ and $\mathrm{SiO}$ ) can explain quite accurately the observed overall spot distribution of the well observed $\mathrm{SiO}$ maser transitions $(v=1 J=1-0, v=2$ $J=1-0$, and $v=1 J=2-1$ ). But the fine details of the relative distribution of both $J=1-0$ lines, in particular their remarkable trend to avoid coincidence, still require dedicated theoretical studies, from which we may expect results allowing estimates of the main properties of the density structure in the inner shells around AGB stars.

Our new maps of $\mathrm{SiO}$ emission from $\mathrm{R} \mathrm{Aqr}$, as well as the analysis of the large number of existing high-resolution maps of this source, do not confirm that the inner circumstellar shell around the red component of this binary system is in rotation, as previously proposed by some authors. Rotation kinematics may be a transient or strongly variable phenomenon, perhaps related to the possible binary periastron by 1996 proposed by McIntosh \& Rustan (2007); but note that the periastron date is very controversial, see Hollis et al. (1997, 2001), Gromadzki \& Mikołajewska (2009). We favor, however, that the spot positions and velocities observed in the maps during the last twelve years mainly reveal radial and random movements within the emitting shell.

Nevertheless, we find that the $\mathrm{SiO}$ maser distributions present an axis of symmetry, identified in most of the observation epochs, at $\mathrm{PA} \sim-10^{\circ}$. This direction is perpendicular to the wide nebulosity detected in the visible and roughly parallel to the usually assumed (but uncertain) value of the projection on the plane of the sky of the axis of the binary orbit. We suggest, in view of the well known interaction between the stellar components of symbiotic systems, an association between the SiO symmetry axis observed in R Aqr and that of the binary system, probably due to gravitational effects of the compact companion on the red giant and/or its close environment.

Acknowledgements. We would like to thank all staff members of the VERA stations and the Mitaka correlation center for their assistance in the observations. H.I. was financially supported by Grant-in-Aid for Scientific Research from the Japan Society for the Promotion of Science (20540234). This work has been supported by the the Spanish Ministry of Education $\mathcal{E}$ Science, under grant PCI2005-A7-0246.

\section{References}

Boboltz, D. A., Diamond, P. J., \& Kemball, A. J. 1997, ApJ, 487, L147 Bujarrabal, V., \& Nguyen-Q-Rieu 1981, A\&A, 102, 65 Bujarrabal, V., \& Pardo, J. R. 2007, Baltic Astron., 16, 126

Bujarrabal, V., Planesas, P., \& del Romero, A. 1987, A\&A, 175, 164 Bujarrabal, V., Alcolea, J., Sanchez Contreras, C., \& Colomer, F. 1996, A\&A, 314,883

Cotton, W. D., Mennesson, B., Diamond, P. J., et al. 2004, A\&A, 414, 275 Cotton, W. D., Vlemmings, W., Mennesson, B., et al. 2006, A\&A, 456, 339 Desmurs, J. F., Bujarrabal, V., Colomer, F., \& Alcolea, J. 2000, A\&A, 360, 189 Gonidakis, I., Diamond, P. J., \& Kemball, A. J. 2006, Recent Advances in Astronomy and Astrophysics, AIP Conf. Proc., 848, 333

Groenewegen, M. A. T., Baas, F., Blommaert, J. A. D. L., et al. 1999, A\&AS, 140,197

Gromadzki, M., \& Mikołajewska, J. 2009, A\&A, 495, 931

Hollis, J. M., Wright, M. C. H., Welch, W. J., et al. 1990, ApJ, 361, 663

Hollis, J. M., Pedelty, J. A., \& Lyon, R. G. 1997, ApJ, 482, L85

Hollis, J. M., Vogel, S. N., van Buren, D., et al. 1999, ApJ, 522, 297

Hollis, J. M., Pedelty, J. A., Forster, J. R., et al. 2000, ApJ, 543, L81

Hollis, J. M., Boboltz, D. A., Pedelty, J. A., White, S. M., \& Forster, J. R. 2001, ApJ, 559, L37

Honma, M., Kijima, M., Suda, H., et al. 2008a, PASJ, 60, 935

Honma, M., Tamura, Y., \& Reid, M. J. 2008b, PASJ, 60, 951

Ivison, R. J., Seaquist, E. R., \& Hall, P. J. 1994, MNRAS, 269, 218

Ivison, R. J., Seaquist, E. R., \& Hall, P. J. 1995, Ap\&SS, 224, 255

Kamohara, R., Bujarrabal, V., Bushimata, T., et al. 2008, PASJ, 60, 1023

Kellogg, E., Anderson, C., Korreck, K., et al. 2007, ApJ, 664, 1079

Lockett, P., \& Elitzur, M. 1992, ApJ, 399, 704

Matsumoto, N., Omodaka, T., Imai, H., et al. 2008, PASJ, 60, 1039

McIntosh, G. C., \& Rustan, G. 2007, AJ, 134, 2113

Michalitsianos, A. G., Oliversen, R. J., Hollis, J. M., et al. 1988, AJ, 95, 1478

Mikołajewska, J. 2007, Baltic Astron., 16, 1

Nakagawa, A., Tsushima, M., Ando, K., et al. 2008, PASJ, 60, 1013 Olofsson, H., Hjalmarson, A., \& Rydbeck, O. E. H. 1981, A\&A, 100, L30

Pardo, J. R., Alcolea, J., Bujarrabal, V., et al. 2004, A\&A, 424, 145

Ragland, S., Le Coroller, H., Pluzhnik, E., et al. 2008, ApJ, 679, 746 
Reid, M. J., \& Menten, K. M. 2007, ApJ, 671, 2068

Rioja, M. J., Dodson, R., Kamohara, R., et al. 2008, PASJ, 60, 1031

Soria-Ruiz, R., Alcolea, J., Colomer, F., et al. 2004, A\&A, 426, 131

Soria-Ruiz, R., Colomer, F., Alcolea, J., et al. 2005, A\&A, 432, L39

Soria-Ruiz, R., Alcolea, J., Colomer, F., Bujarrabal, V., \& Desmurs, J.-F. 2007, A\&A, 468, L1

\section{Appendix A: Annual parallax measurement from December 2004 to March 2006}

Though the fitting of the spot distribution observed in August 2006, Fig. 8, is not very bad, the obtained radius is too small compared with those of other epochs, and the distribution of the spots around the star is far from a circle. Accordingly, we also estimated the annual parallax and proper motions not taking the measurement obtained on 30 Aug. 2006 into account, which can be considered as a further test of the accuracy of our measurements. We find $\pi=3.5 \pm 0.4$ mas, yielding a distance to R Aqr $D=284_{-29}^{+36} \mathrm{pc}$. This value is consistent with our best result, obtained by also taking this epoch into account, see Sect. 3, and even with the Hipparcos parallax, mainly because of the large uncertainty of the Hipparcos measurement. We show the residuals from the best-fit curve in Figs. A.1 and A.2.

Subtracting the parallax movements, we also estimated the linear proper motions in RA and declination. We obtain $\mu_{\mathrm{RA}}=$

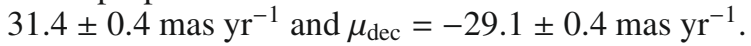

We finally note that if we take our eight observations into account, even the uncertain fitting of the data obtained in October 2006, we obtain $\pi=4.7 \pm 0.9$ mas, $\mu_{\mathrm{RA}}=32.7 \pm$ $0.8 \mathrm{mas} \mathrm{yr}^{-1}$ and $\mu_{\mathrm{dec}}=-30.2 \pm 0.7 \mathrm{mas} \mathrm{yr}^{-1}$. (These values are very close to those obtained with our standard method, Sect. 3, in which all data except for this last epoch are included.)

\section{Appendix B: Complementary data}

In this Appendix we present complementary data from our observations.

Figures B.1, B.2, and B.3 show our maps obtained in May and July 2006. In these epochs, the maps could not be phasereferenced with respect to the continuum source placed in the second VERA beam, because of technical problems, so we could not obtain absolute coordinates and an accurate comparison of the $v=1$ and $v=2$ spot positions. The relative coordinates are in them given with respect to a bright spot used as reference for the phase calibration. For these observations we do not calculate proper motions and do not give any comparison of the relative position of the $v=1$ and $v=2$ spots (see Table 1). But they are useful for our discussion of the shape and dynamics of the inner circumstellar shells and of their variations with time (Sects. 5.1, 5.2).

To present more in detail our results, we finally present maps per velocity channels obtained from our other observations (in which absolute coordinates were measured) in Figs. B.4 to B.9, corresponding to the maps shown in Figs. 2 to 9.

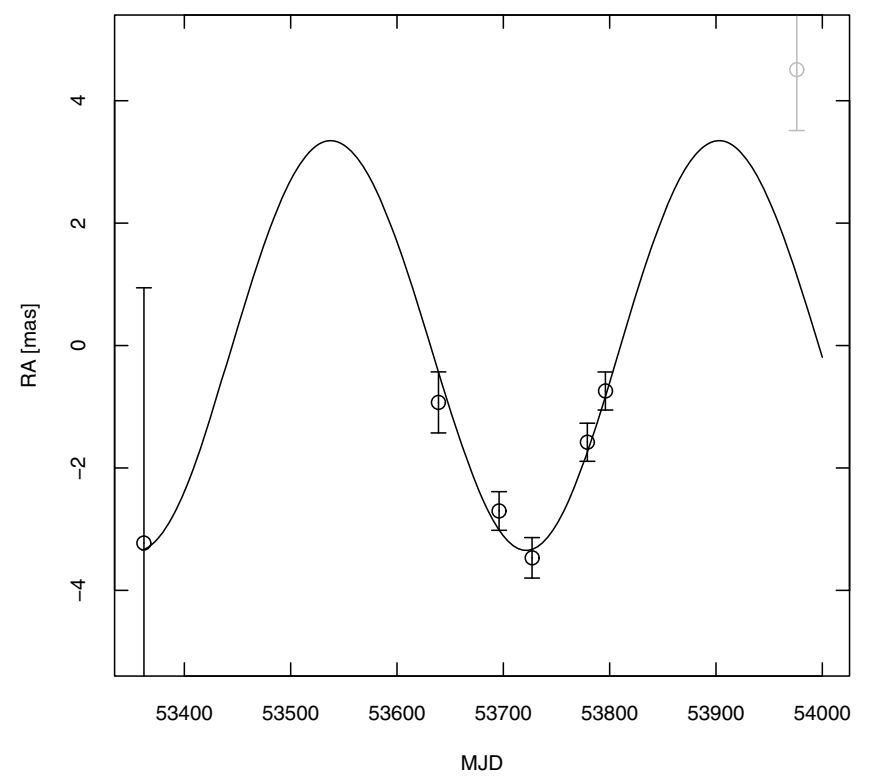

Fig. A.1. Relative right ascension of the $\mathrm{SiO}$ maser centroid measured in R Aqr, after subtracting the linear proper motion, and the parallax movement fitted to the data not taking into account the observations obtained in August 2006.

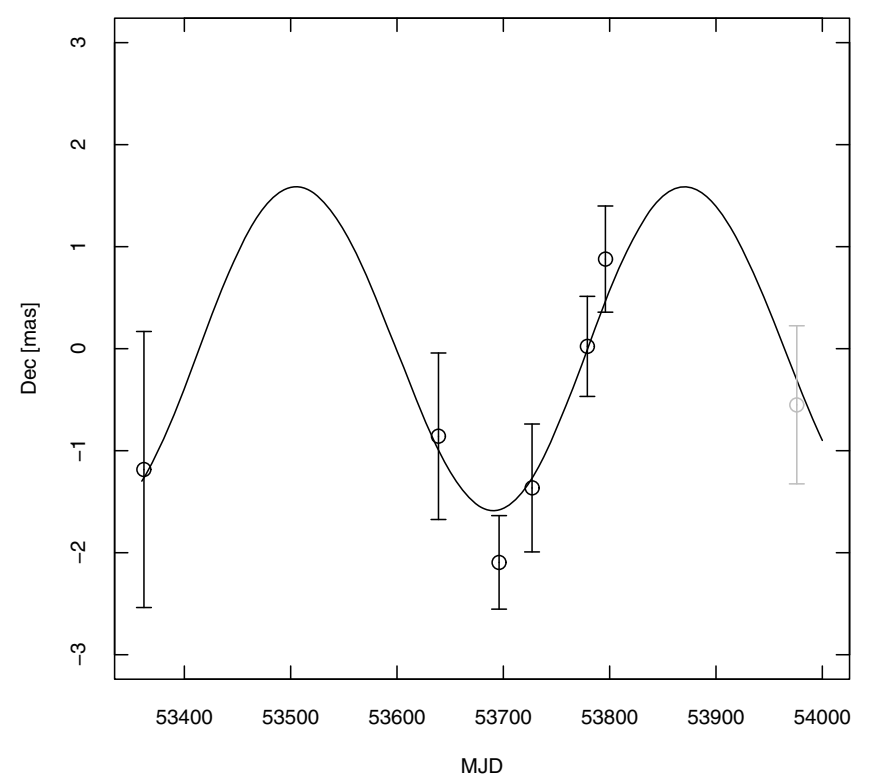

Fig. A.2. Relative declination of the $\mathrm{SiO}$ maser centroid measured in $\mathrm{R}$ Aqr, after subtracting the linear proper motion, and the parallax movement fitted to the data not taking into account the observations obtained in August 2006. 
A\&A 510, A69 (2010)
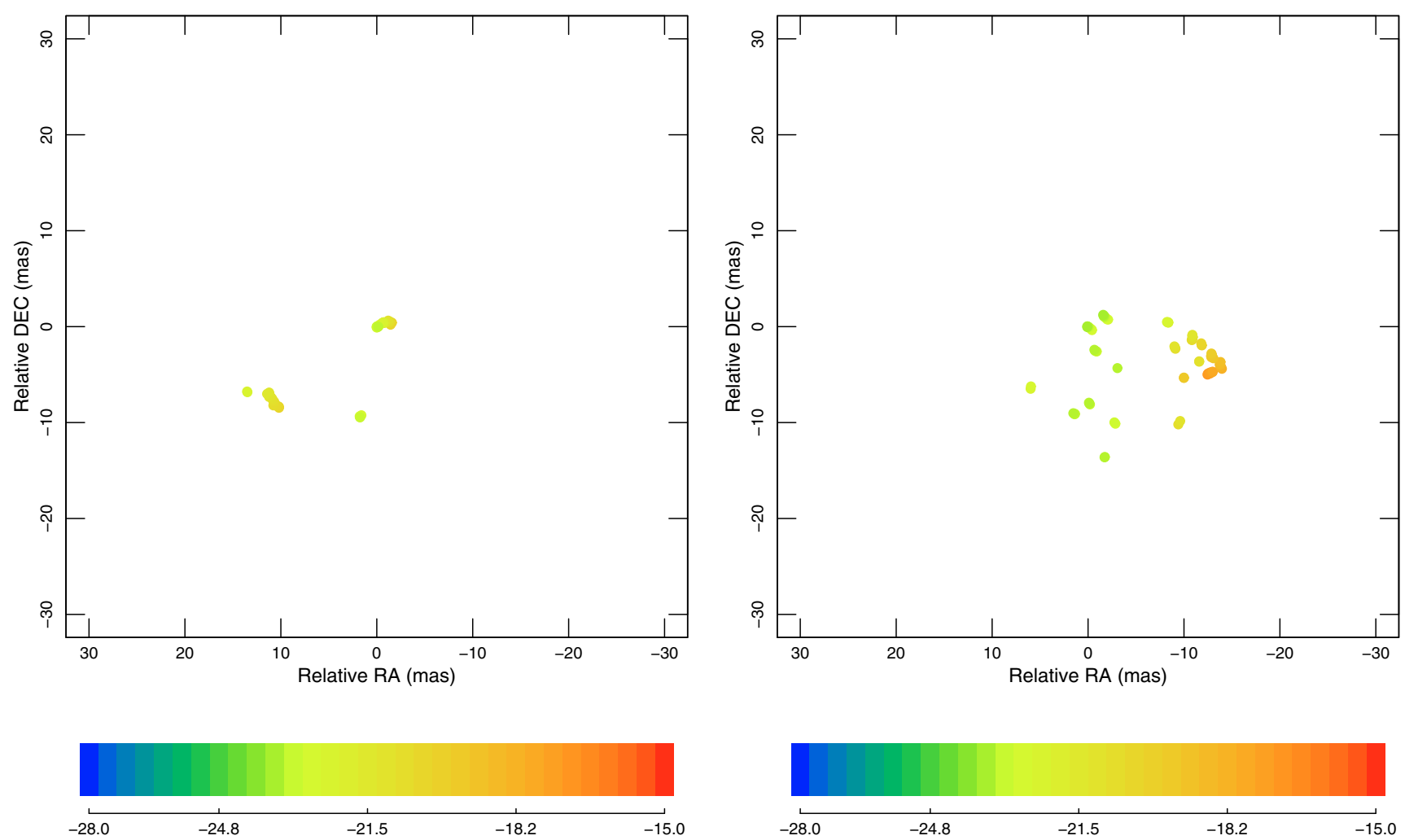

Fig. B.1. Maps of $v=1$ (left panel) and $v=2$ (right panel) $J=1-0$ obtained on 8 May 2006. The colors indicate the $L S R$ velocity. The relative positions are given with respect to one of the spots.
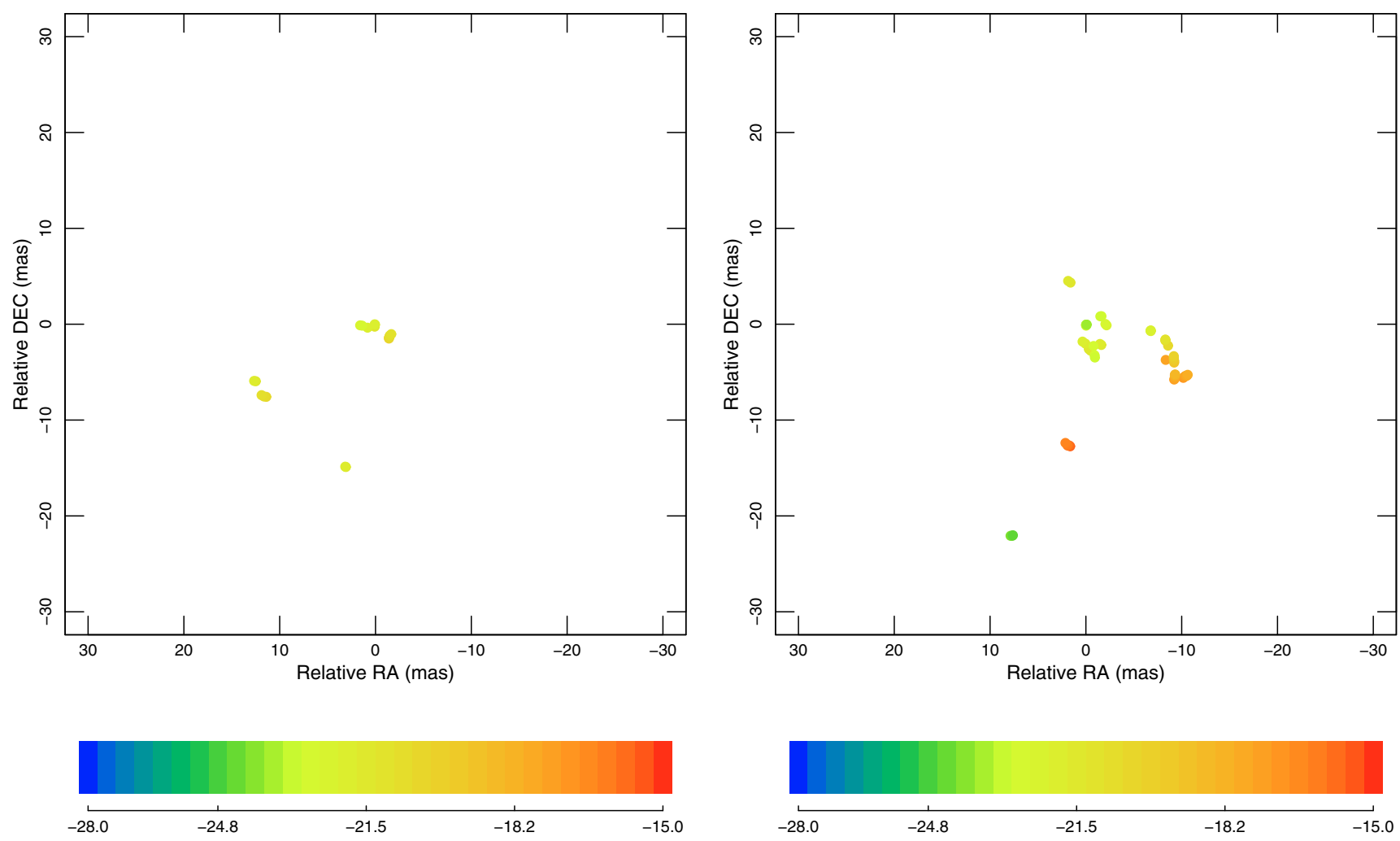

Fig. B.2. Maps of $v=1$ (left panel) and $v=2$ (right panel) $J=1-0$ obtained on 11 July 2006 . The colors indicate the LSR velocity. The relative positions are given with respect to one of the spots. 
R. Kamohara et al.: VERA observations of $\mathrm{SiO}$ maser emission from R Aquarii
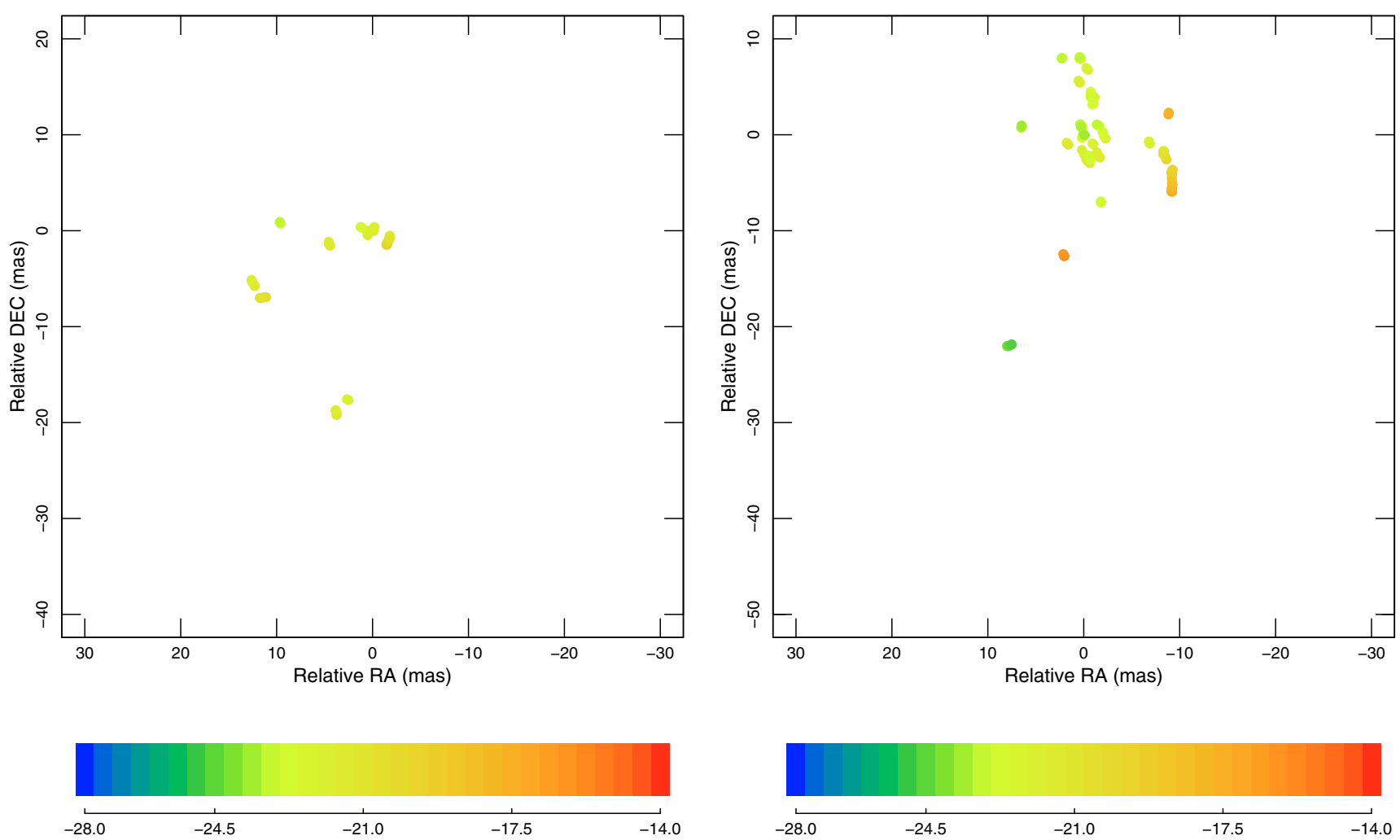

Fig. B.3. Maps of $v=1$ (left panel) and $v=2$ (right panel) $J=1-0$ obtained on 26 July 2006. The colors indicate the LSR velocity. The relative positions are given with respect to one of the spots.
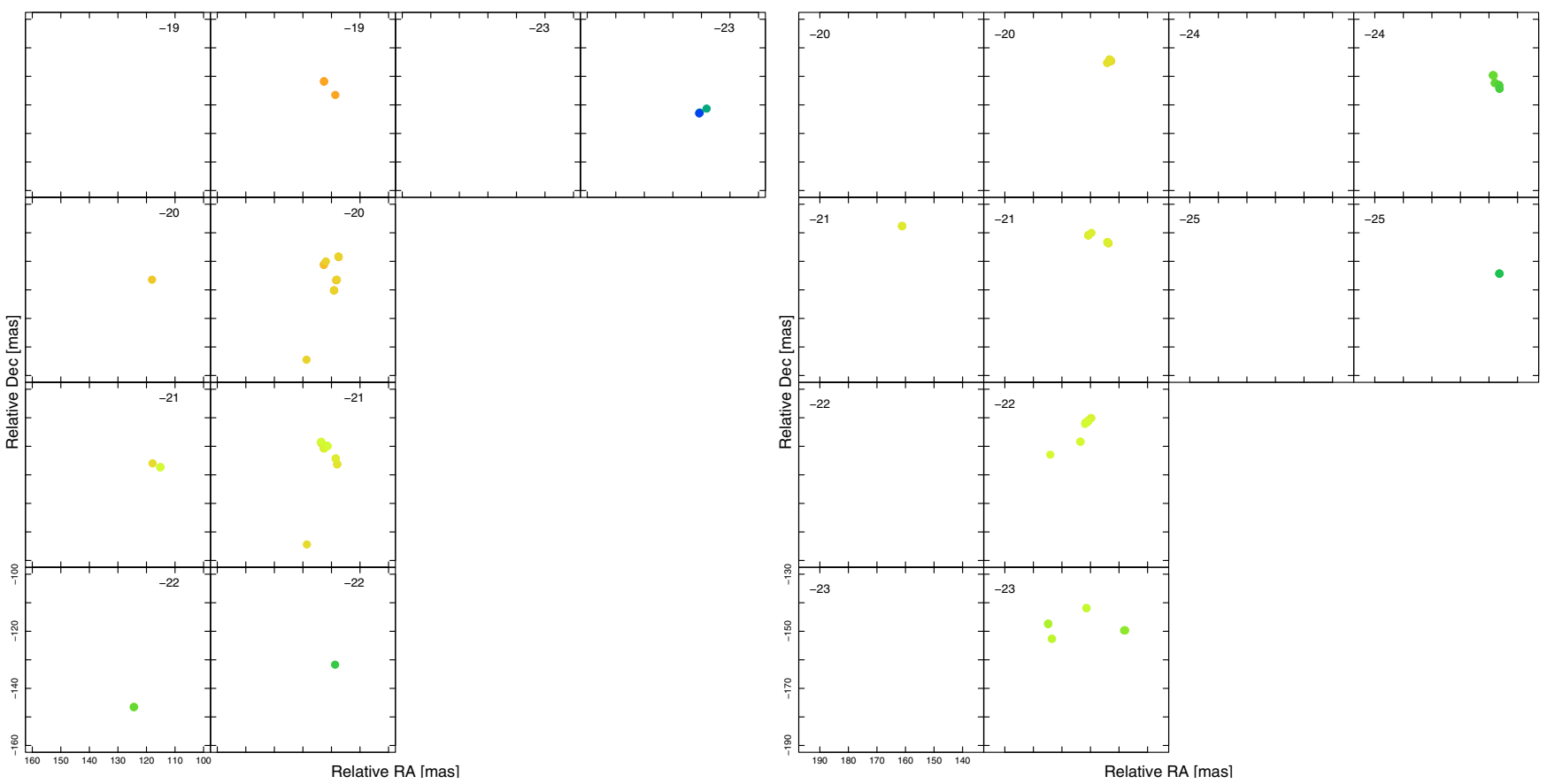

Fig. B.4. Maps per velocity channel obtained on 23 December 2004 (left) and 27 September 2005 (right). The LSR velocity of each channel is shown in the panels. In each epoch, the left columns show the $v=1 J=1-0$ maser spots, and the right columns show the $v=2 J=1-0$ spots. 
A\&A 510, A69 (2010)
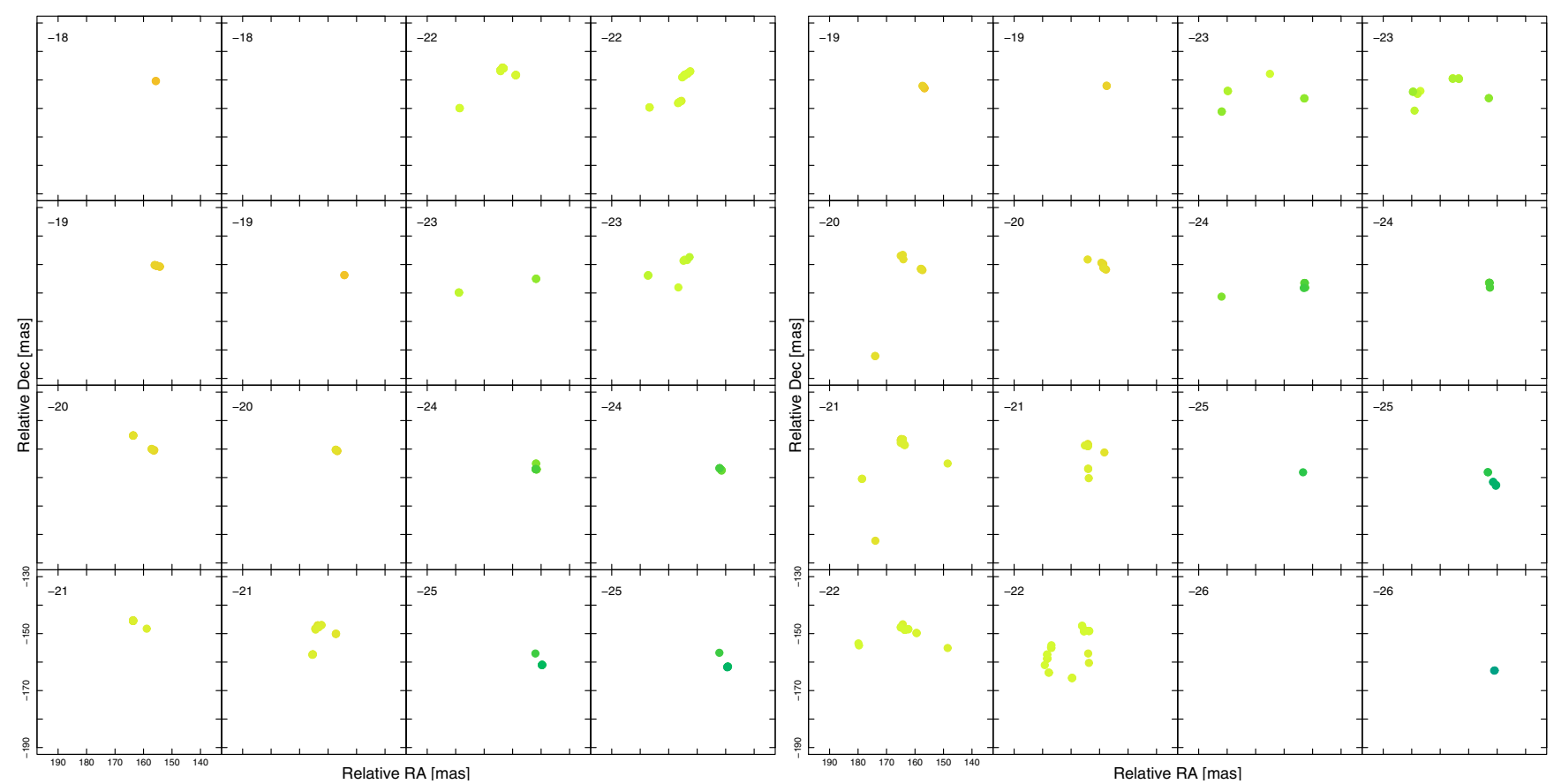

Fig. B.5. Same as Fig. B.4, for the maps obtained on 23 November 2005 (left) and 24 December 2005 (right).
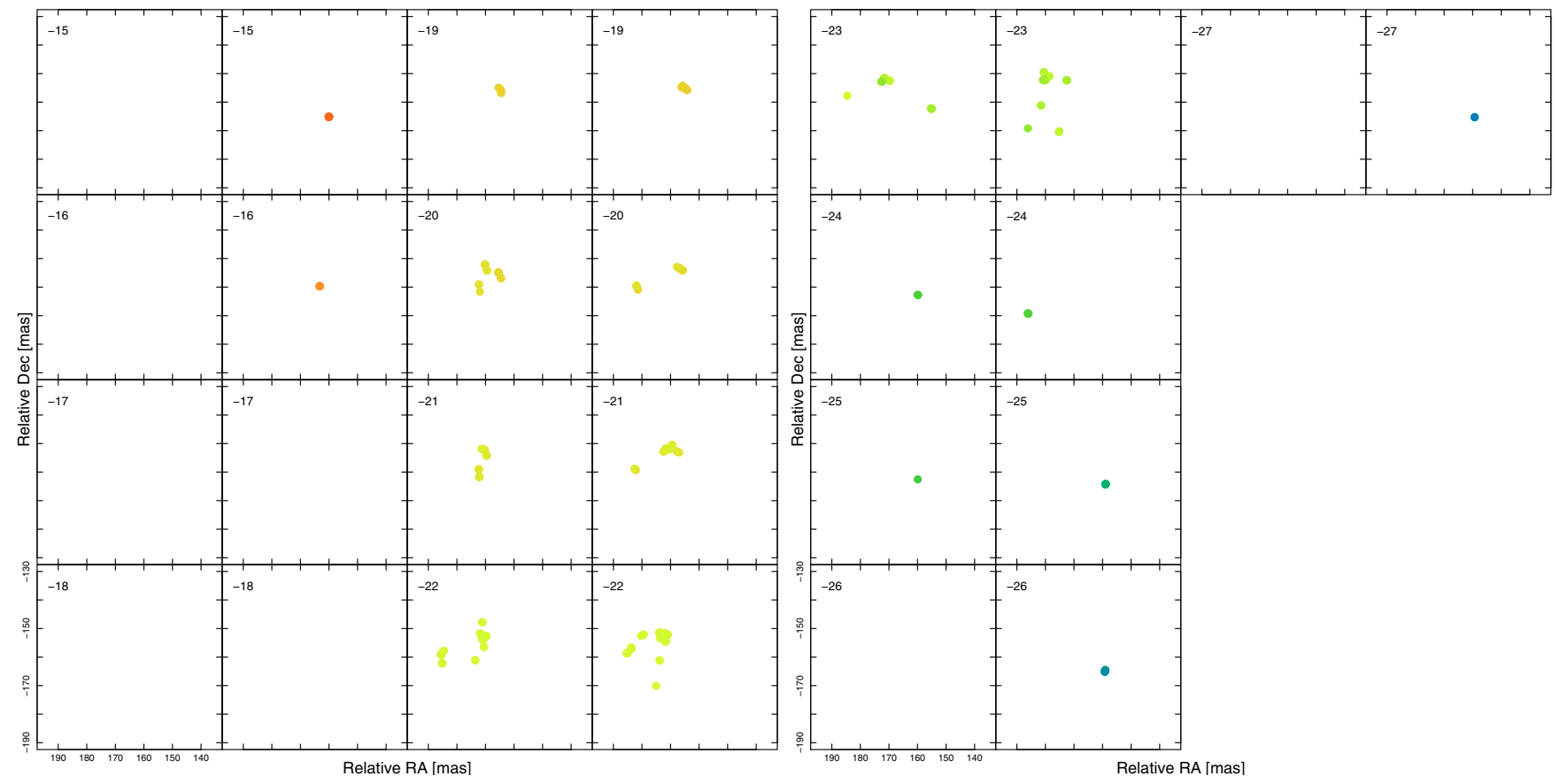

Fig. B.6. Same as Fig. B.4 for the maps obtained on 14 February 2006. 
R. Kamohara et al.: VERA observations of $\mathrm{SiO}$ maser emission from R Aquarii
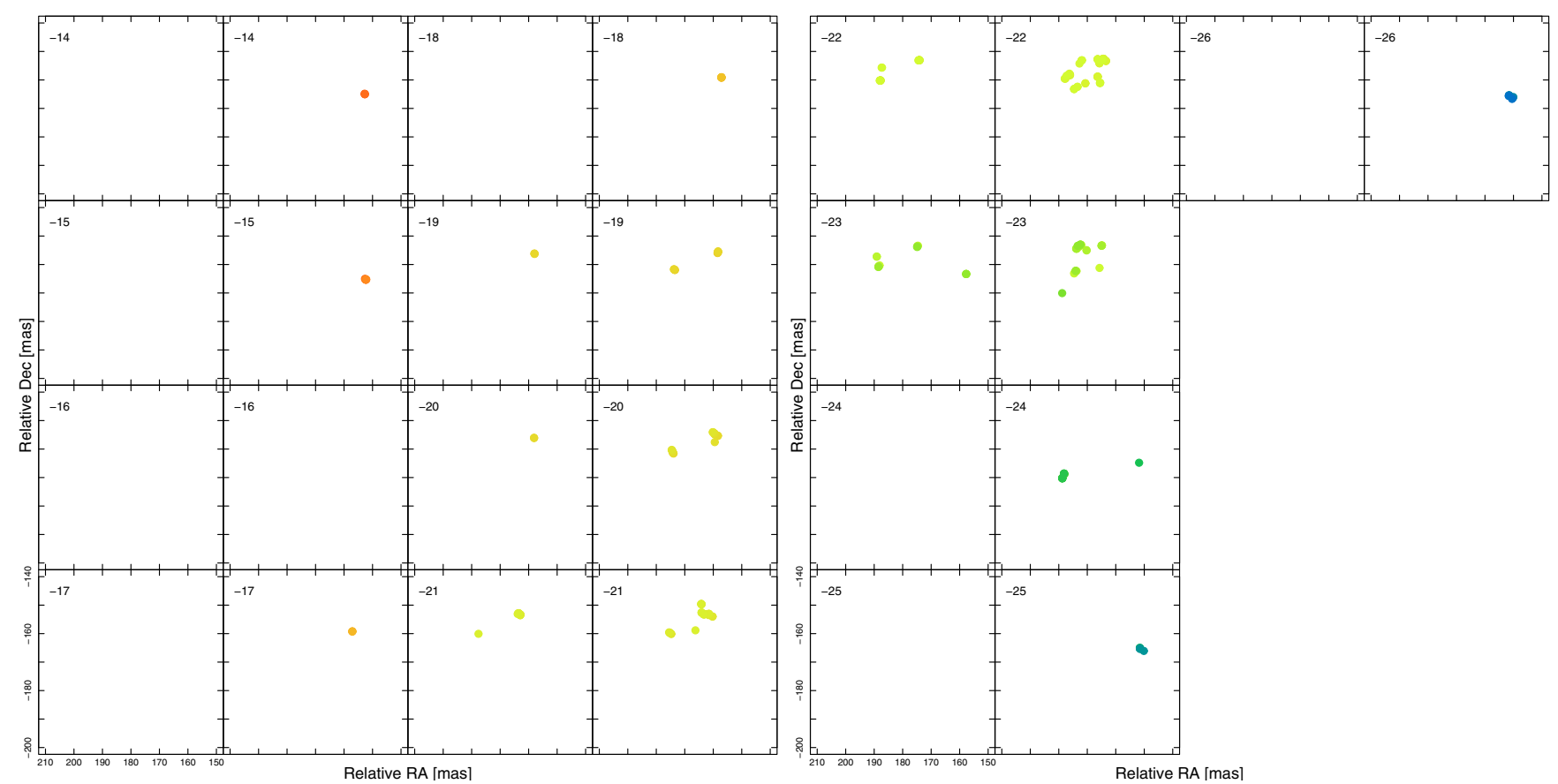

Fig. B.7. Same as Fig. B.4 for the maps obtained on 4 March 2006.

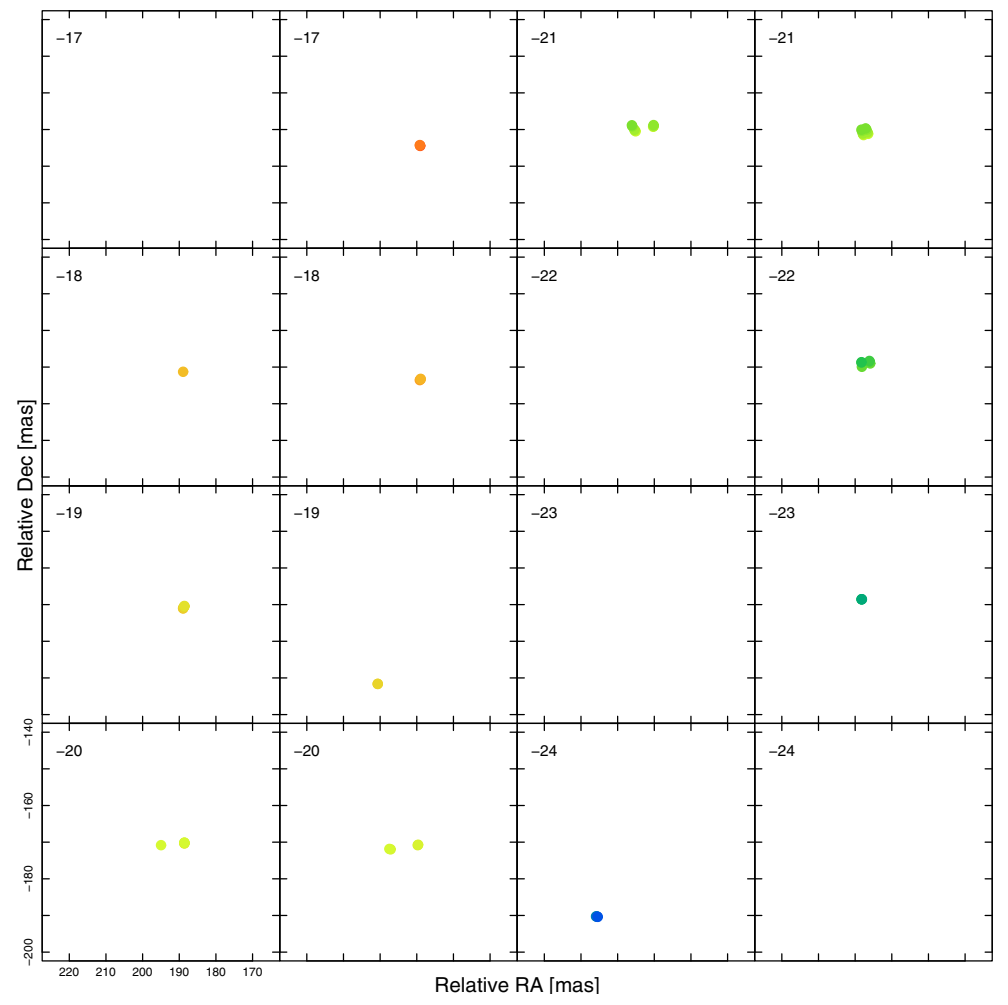

Fig. B.8. Same as Fig. B.4 for the maps obtained on 30 August 2006. 
A\&A 510, A69 (2010)

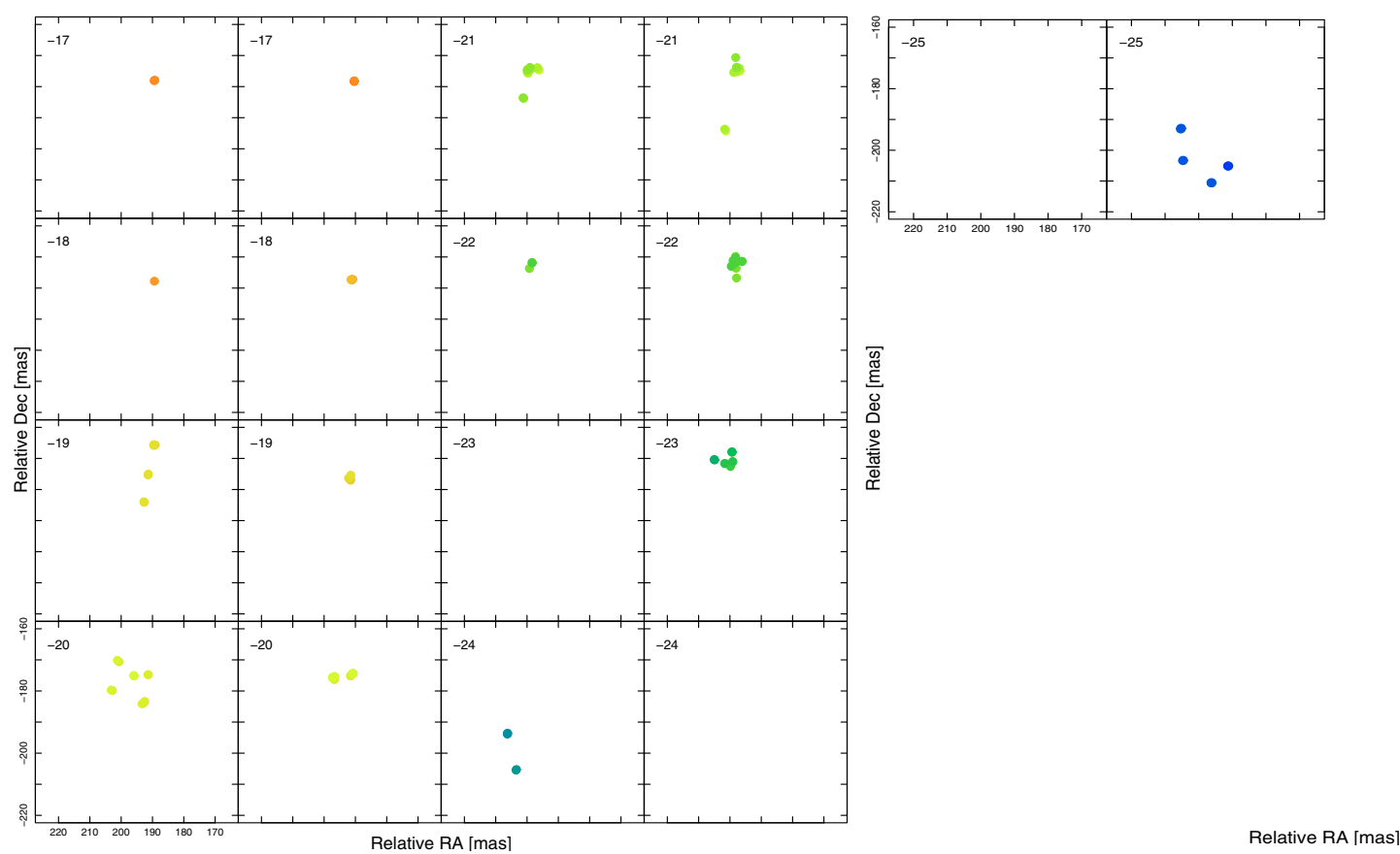

Fig. B.9. Same as Fig. B.4 for the maps obtained on 13 October 2006. 